\title{
Image-based Micro-continuum Model for Gas Flow in Organic-Rich Shale Rock
}

DOI:

10.1016/j.advwatres.2018.10.004

\section{Document Version}

Accepted author manuscript

Link to publication record in Manchester Research Explorer

\section{Citation for published version (APA):}

Guo, B., Ma, L., \& Tchelepia, H. A. (2018). Image-based Micro-continuum Model for Gas Flow in Organic-Rich Shale Rock. Advances in Water Resources, 122. https://doi.org/10.1016/j.advwatres.2018.10.004

\section{Published in:}

Advances in Water Resources

\section{Citing this paper}

Please note that where the full-text provided on Manchester Research Explorer is the Author Accepted Manuscript or Proof version this may differ from the final Published version. If citing, it is advised that you check and use the publisher's definitive version.

\section{General rights}

Copyright and moral rights for the publications made accessible in the Research Explorer are retained by the authors and/or other copyright owners and it is a condition of accessing publications that users recognise and abide by the legal requirements associated with these rights.

\section{Takedown policy}

If you believe that this document breaches copyright please refer to the University of Manchester's Takedown Procedures [http://man.ac.uk/04Y6Bo] or contact uml.scholarlycommunications@manchester.ac.uk providing relevant details, so we can investigate your claim.

\section{OPEN ACCESS}




\section{Accepted Manuscript}

Image-based Micro-continuum Model for Gas Flow in Organic-Rich Shale Rock

Bo Guo, Lin Ma, Hamdi A. Tchelepi

PII: S0309-1708(18)30637-7

DOI: https://doi.org/10.1016/j.advwatres.2018.10.004

Reference: ADWR 3212

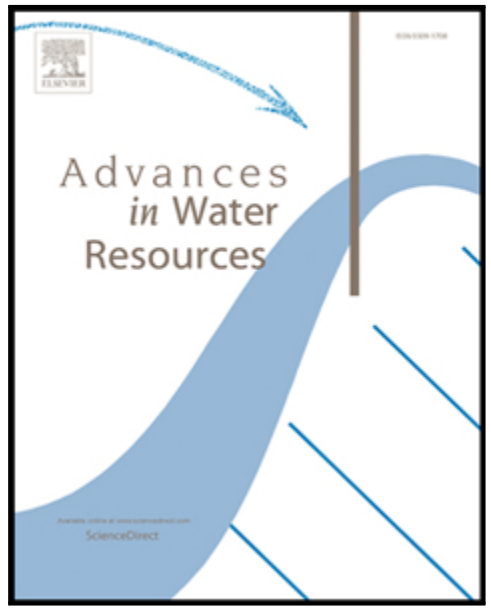

To appear in:

Advances in Water Resources

Received date:

25 July 2018

Revised date:

21 September 2018

Accepted date:

3 October 2018

Please cite this article as: Bo Guo, Lin Ma, Hamdi A. Tchelepi, Image-based Micro-continuum Model for Gas Flow in Organic-Rich Shale Rock, Advances in Water Resources (2018), doi: https://doi.org/10.1016/j.advwatres.2018.10.004

This is a PDF file of an unedited manuscript that has been accepted for publication. As a service to our customers we are providing this early version of the manuscript. The manuscript will undergo copyediting, typesetting, and review of the resulting proof before it is published in its final form. Please note that during the production process errors may be discovered which could affect the content, and all legal disclaimers that apply to the journal pertain. 


\title{
Image-based Micro-continuum Model for Gas Flow in Organic-Rich Shale Rock
}

\author{
Bo Guo ${ }^{\mathrm{a}, *}$, Lin Ma ${ }^{\mathrm{b}, \mathrm{c}}$, Hamdi A. Tchelepi ${ }^{\mathrm{a}}$ \\ ${ }^{a}$ Department of Energy Resources Engineering, Stanford University, Stanford, California, USA \\ ${ }^{b}$ School of Earth, Atmospheric, and Environmental Sciences, University of Manchester, Manchester, UK \\ ${ }^{c}$ Manchester X-ray Imaging Facility, School of Materials, University of Manchester, Manchester, UK
}

\begin{abstract}
The physical mechanisms that control the flow dynamics in organic-rich shale are not well understood. The challenges include nanometer-scale pores and multiscale heterogeneity in the spatial distribution of the constituents. Recently, digital rock physics (DRP), which uses high-resolution images of rock samples as input for flow simulations, has been used for shale. One important issue with images of shale rock is sub-resolution porosity (nanometer pores below the instrument resolution), which poses serious challenges for instruments and computational models. Here, we present a micro-continuum model based on the Darcy-Brinkman-Stokes framework. The method couples resolved pores and unresolved nano-porous regions using physics-based parameters that can be measured independently. The Stokes equation is used for resolved pores. The unresolved nano-porous regions are treated as a continuum, and a permeability model that accounts for slip-flow and Knudsen diffusion is employed. Adsorption/desorption and surface diffusion in organic matter are also accounted for. We apply our model to simulate gas flow in a high-resolution 3D segmented image of shale. The results indicate that the overall permeability of the sample (at fixed pressure) depends on the time scale. Early-time permeability is controlled by Stokes flow, while the late-time permeability is controlled by
\end{abstract} non-Darcy effects and surface-diffusion.

Keywords: Shale gas, nanoporous media, micro-continuum, Darcy-Brinkman-Stokes

\section{Introduction}

Oil and gas production from unconventional subsurface resources, such as ultra-tight organic-rich shales, 3 has increased significantly in the past decade. Shale gas now accounts for more than half of the gas production 4 in the United States [1]. Despite the rapid development of the shale gas industry, it remains challenging to 5 predict and further enhance gas production from shale formations. The majority of gas in a shale formation

6 is stored in the rock matrix - primarily in organic matter $[2,3]$. During production, the stored gas has to 7 travel through the matrix to reach natural and hydraulic fractures that provide pathways to the production 8 well. Gas transport in the shale matrix is therefore one of the most critical processes for production.

\footnotetext{
* Corresponding author. Now at the Department of Hydrology and Atmospheric Sciences, University of Arizona.

Email address: boguo@email.arizona.edu (Bo Guo)
} 
Understanding gas transport is also critical when considering using the depleted shale gas reservoir for carbon dioxide storage [4]. However, due to the complex pore structure and heterogeneity of the shale materials, gas transport mechanisms in the shale matrix are not well understood.

The shale rock matrix is strongly heterogeneous at multiple length scales in terms of pore structures and the material constituents, both of which may dictate gas transport in shale. The range of pore sizes in shale can be assessed using indirect petrophysical methods $[5,6,7]$, such as mercury injection capillary pressure (MICP), low pressure nitrogen adsorption, and nuclear magnetic resonance (NMR) spectroscopy. The measurements suggest that the majority of the pore sizes of shale are on the order of a few to tens of nanometers. In addition to the indirect measurements, several imaging techniques have been used to directly characterize shale samples in three dimensions (3D), such as X-ray computed tomography (e.g., micro-CT, nano-CT), focused ion beam scanning electron microscopy (FIB-SEM), helium ion microscopy (HIM), and transmission electron microscopy (TEM) tomography. Image characterizations often aim to understand the type, geometry, size, surface area, and connectivity of the pores, the distribution of material constituents, as well as the relationships between pores and constituents [8,6,9]. 3D shale images confirm the nanometer range of pores in shale inferred from indirect methods [8]. Kelly et al. [10] assessed the utility of shale FIB-SEM images and concluded that FIB-SEM images may not provide a representative elementary volume (REV) for shale permeability. Limited by a trade-off between the image resolution and the field of view, multiple imaging techniques with different inherent resolutions may need to be combined to characterize larger shale samples $[11,12,13]$. Wu et al. [13] used micro-CT, nano-CT, FIB-SEM, and HIM to characterize a shale sample of $1.3^{3} \mathrm{~cm}^{3}$ and subsamples down to $3.25^{3} \mu \mathrm{m}^{3}$. The highest resolution images obtained using HIM indicate that pores, which appeared to be isolated in lower resolution FIB-SEM images, are connected by smaller pores. Ma et al. [11] and Ma et al. [12] used micro-CT, nano-CT, and FIB-SEM to address the issue of an REV for porosity and different material constituents. Their highest resolution FIB-SEM images were segmented into four material constituents: macro-pores, organic matter, clay minerals, and granular minerals. We note that pores are commonly categorized as micropores $(<2 \mathrm{~nm})$, mesopores $(2-50 \mathrm{~nm})$, and macro-pores (> $50 \mathrm{~nm})$ [14]. Here, we use a different nomenclature and refer to the pores that are resolved in the image as macro-pores and pores that are below the image resolution as sub-resolution pores. Figure 1 shows an example of a 3D FIB-SEM image from Ma et al. [12] for a shale sample from the Haynesville-Bossier shale formation. The 3D digital images show the complexity of the pore space of shale. The image in Figure 1 is used in our pore-scale simulation study (more details of the image are presented in section 4 ).

The nanometer-range pores in shale rock leads to many interesting physical processes that deviate from the standard Darcy's law for flow in porous media, or the standard formulation of the Navier-Stokes equations at the pore-scale. This dates back to the work of Klinkenberg [15] who reported that gas permeability of low permeable tight rocks is larger than their intrinsic permeability (e.g., measured with liquids), especially at low gas pressure. The Klinkenberg effect can be attributed to a non-zero slip velocity at the pore wall. Gas 
flow in small confined pores belongs to the subject of rarefied gas dynamics. Small confined pores lead to gas rarefaction where collisions between pore wall and gas molecules become important relative to the collisions between gas molecules. The extent of the rarefaction effect can be characterized by the Knudsen number $(\mathrm{Kn})$, which is defined as the ratio between the mean free path to the characteristic geometric length of the flow conduit. Gas flow can be classified into four regimes [16]: continuum flow (Kn $\lesssim 0.001)$ where NavierStokes equations are applicable; slip flow $(0.001<\mathrm{Kn} \lesssim 0.1)$ in which the Navier-Stokes equations can still be applied, but with appropriate velocity slip conditions at the solid surface; transition flow $(0.1<\mathrm{Kn} \lesssim 10)$ where gas flow transits from slip flow to free molecular flow; free molecular flow $(\mathrm{Kn}>10)$ where the continuum-based Navier-Stokes equations are not applicable. Note that this classifieation is based on gas flow through an infinite channel with the thickness being the characteristic length. The regime boundaries (in terms of $\mathrm{Kn}$ ) depend on the specific geometries of the conduits. Beskok and Karniadakis [17] developed a unified model for rarefied gas flow in micro-channels, ducts, and pipes. Their/model is applicable for all flow regimes $(0 \leqslant \mathrm{Kn}<\infty)$. We refer to the unified model as the BK model. Wu and Zhang [18] extended the BK model to include the effects of adsorption in the nano-channel accounting for migration of the adsorbed phase. Civan [19] used the BK model to develop an apparent permeability for tight rocks using the bundle-of-tubes model, which was further extended to include surface diffusion [20]. Lunati and Lee [21] developed a bundle-of-dual-tubes model for gas production from fractured shale formations. In the spirit of the dusty-gas model [22], another group of apparent permeability models has been derived by a linear, or weighted, summation of the flux contributions from viscous flow, Knudsen diffusion, and surface diffusion [see 23, 24, 25, 26]. Landry et al. [27] compared the BK model, the dusty-gas model, and the Javadpour [23] model with the linearized Boltzmann solution for a straight tube. They reported that both the dustygas and the Javadpour [23] models overestimate the permeability, while the BK model matches well with the linearized Boltzmann solution. In addition, Landry et al. [27] showed that the bundle-of-tubes model can significantly overestimate the apparent permeability due to flow dependence on the pore shapes and connectivity in the slip and early-transition flow regimes. To date, the apparent permeability models in the literature are either limited to idealized porous media (i.e., bundle-of-tubes with correction for tortuousity) or composite porous media with a presumed distribution of constituents that allow for upscaling [24, 28]. The appropriate 'apparent' permeability and other transport properties of shale rocks remain open questions. Images of shale samples provide an opportunity to model the complex transport dynamics directly for natural shale samples. This should provide insight into the complex physics associated with transport in naturalshales. The use of pore-scale imaging and numerical flow modeling, referred to as digital rock physics or analysis, is increasing for conventional rocks [29, 30]. For unconventional source rocks (i.e., organic-rich shale), image-based pore-scale modeling is still in its infancy. Lattice Boltzmann methods (LBM), as a popular method for pore-scale simulation, has been used for direct numerical simulation of shale gas transport [31]. Various studies have extended LBM to include the transport physics relevant to shale, e.g., Knudsen diffusion, slip flow, adsorption/desorption and surface diffusion [e.g., 32, 33]. Another approach is to solve 
the Navier-Stokes equations in the pore structures using finite-volume discretization schemes. Because most of the pores are in the nanometer range, the applicability of the standard Navier-Stokes equations may be questioned. In addition, many pores with sizes in the nanometer range cannot be resolved due to the limitation of image resolution. These sub-resolution pores are critical for gas transport as the important nanoscale physics (e.g., slip flow, Knudsen diffusion, adsorption/desorption, and surface diffusion) primarily take place in those unresolved nano-porous regions. Therefore, it is crucial to consider the sub-resolution pores and include the nanoscale transport physics.

For conventional rocks, a micro-continuum modeling framework has been developed to address the subresolution porosity in micro-CT images [34]. Such micro-continuum approaches consider the unresolved porous region (pore sizes below image resolution) as a continuum, and use Darcy's law for the fluid flow. The Stokes equation is used for the resolved pores and is coupled with the Darcy equation in the unresolved porous region based on the Darcy-Brinkman-Stokes equations [35]. The porosity $(\phi)$ is used as an indicator to differentiate void space from the unresolved porous region $(\phi=1$, represents void, $0<\phi<1$ represents sub-resolution porous region). Similar multiscale concepts have also been developed for pore network models to address the subresolution microporosity $([36],[37])$. In the present work, we develop a micro-continuum modeling framework to model gas transport in organic-rich shale based on 3D digital images (obtained from micro-CT, nano-CT, or FIB-SEM; FIB-SEM images are used in our work). For pores that are resolved fully in the image, we model the gas flow with the Stokes equation. The unresolved regions, where the pore sizes are below the image resolution, are treated as a continuum (porous medium). For this nanoporous subresolution continuum, we develop an apparent permeability model that accounts for non-Darcy effects (i.e., slip flow and Knudsen diffusion), adsorption/desorption, and surface diffusion. The specific model for the nano-porous region depends on the types of material constituents segmented from the image (see Figure 1). Here, the nano-porous region includes organic matter and clay; adsorption/desorption and surface diffusion are accounted for in the organic matter, but not in clay. The granular minerals, consisting of quartz, calcite, ankerite, albite, and pyrite for the sample we use, have negligible porosity and are considered impermeable $[12]$.

The paper is organized as follows. In section 2 we introduce the gas transport mechanisms and summarize current models for shale gas transport. Then, the micro-continuum framework is introduced. We apply the model to simulate a 3D FIB-SEM image (a subset of the image in Figure 1) to compute the apparent permeability. We then simulate the transient gas production process. Analysis of the numerical experiments is presented in section 4 . Then, we discuss the implications of the analysis, the limitations of the model, and future directions in section 5 . We close with concluding remarks in section 6 .

\section{Physics and modeling of shale gas transport}

Gas transport in shale differs from that in conventional formations due to the extremely small (nanometer range) pores and the strong heterogeneity of organic-rich shale materials. Different mechanisms dominate 


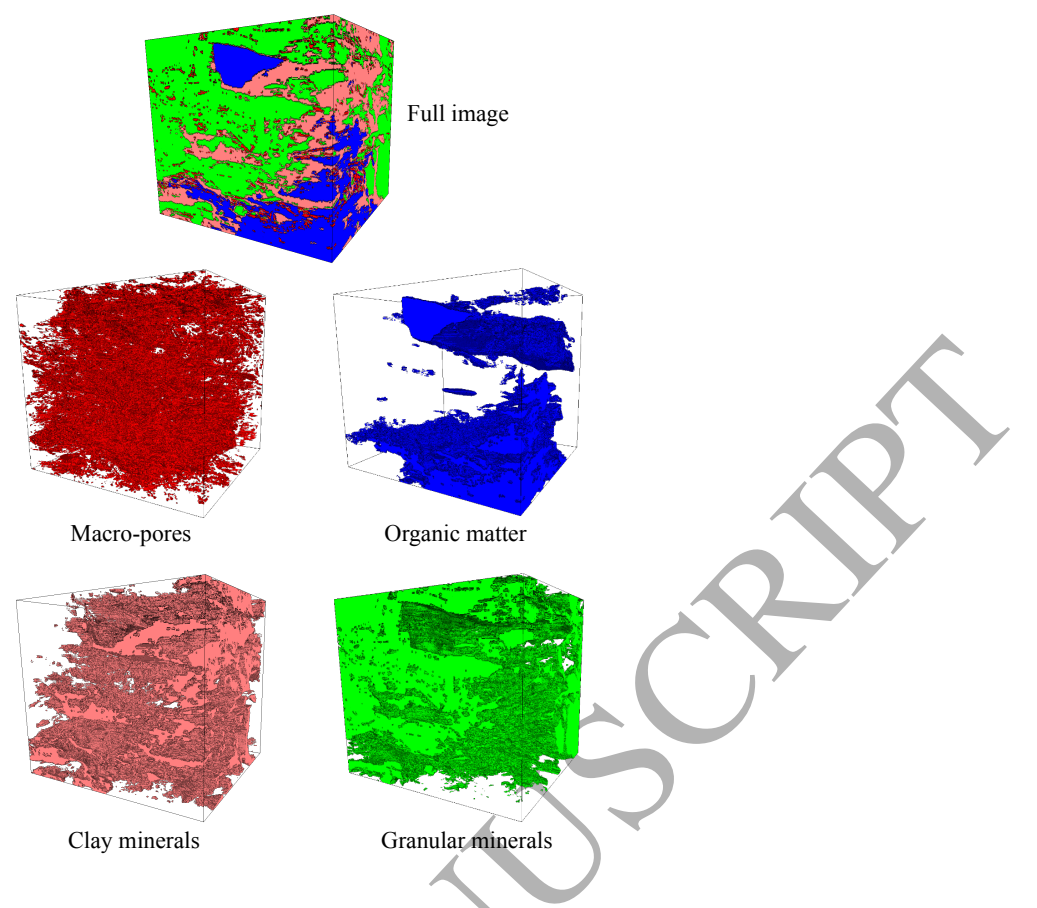

Figure 1: 3D shale image reconstructed from FIB-SEM [12]. The size of the image is: $7.22 \mu \mathrm{m} \times 5.11 \mu \mathrm{m} \times 6.82 \mu \mathrm{m}$; voxel size: $10 \mathrm{~nm} \times 10 \mathrm{~nm} \times 20 \mathrm{~nm}$. Four material constituents are segmented: red - macro-pores; blue - organic matter; pink - clay minerals; green - granular minerals. The shale sample is from a core in the Haynesville-Bossier shale formation.

the transport in different materials. Thus, models with different physics need to be applied to different material constituents of shale rock. In this section, we summarize the models for gas transport in complex shale material constituents. Note that our paper considers only a single-component - methane, and the term 'gas' refers to methane.

\subsection{Gas flow through straight tubes and idealized porous media}

We summarize models for the rarefied gas flow in straight tubes with radial and rectangular cross-sections, and then extend the simple tube models to gas flow in idealized nano-porous media using the bundle-of-tubes representation.

\subsubsection{Equation-of-state for free methane}

The density and viscoity of methane vary with pressure and temperature. The density of free methane $\left(\rho_{f}\right)$ can be expressed as

$$
\rho_{f}=\frac{p_{f} M}{Z R T},
$$

where $p_{f}$ is the pressure of free methane, $M$ is the methane molecular weight, $Z$ is the compressibility factor which equals to unity for ideal gas, $R$ is the gas constant, and $T$ is the absolute temperature. The subscript ' $f$ ' denotes free gas. 
while for a rectangular tube, the correction factor is

$$
f(\mathrm{Kn})=(1+\alpha \mathrm{Kn})\left(1+\frac{6 \mathrm{Kn}}{1-b \mathrm{Kn}}\right)
$$


where $\mathrm{Kn}=\lambda / r$ for a radial tube with $r$ being the radius; $\mathrm{Kn}=\lambda / h$ for a rectangular tube with $h$ being the height; $C_{r}(\mathrm{Kn})=1+\alpha \mathrm{Kn}$ is the rarefaction coefficient that models the effect of reduced inter-molecular collisions with increased $\mathrm{Kn} ; \alpha$ is an coefficient that can be determined by experiments or from linearized Boltzmann solution; $b$ is the slip coefficient and is determined as $b=-1$ for tube flow representing a second-order slip condition. For a radial tube, $q_{\mathrm{H}-\mathrm{P}, f}=\pi r^{4} / 8 / \mu_{f}$, while for a rectangular tube, $q_{\mathrm{H}-\mathrm{P}, f}=$ $C(\mathrm{AR}) w h^{3} / 12 / \mu_{f}$ where $w$ is the width of the rectangular cross-section and $C(\mathrm{AR})$ is the correction factor for the geometry that is a function of the aspect ratio of the rectangular channel $(\mathrm{AR}=w / h)$ and is independent of $\mathrm{Kn}$. Note that $f(\mathrm{Kn}) \rightarrow 1$ as $\mathrm{Kn} \rightarrow 0$, and $q_{f}$ recovers the Hagen-Poiseuille flux.

By comparing the volumetric flux predicted by Equations (5) and (6) with the linearized Boltzmann solution, $\alpha$ is fitted as an empirical analytical function $\alpha=\alpha_{0}(2 / \pi) \tan ^{-1}\left(\alpha_{1} \mathrm{Kn}^{\beta}\right)$ with two free parameters $\alpha_{1}$ and $\beta . \alpha_{0}$ is determined by equating the flux predicted from Equations (5) and (6) to the free molecular rate (Knudsen diffusion) when $\mathrm{Kn} \rightarrow \infty$.

The BK model works well for rarefied gas flow in straight tubes for all flow regimes $(0 \leqslant \mathrm{Kn}<\infty)$; however, a similar unified model has not been developed for complex natural porous media, which consist of many connected nano-scale pores and pore throats. Rarefied gas flow through porous medium with low permeability (tight rocks) was first systematically studied by Klinkenberg [15]. Klinkenberg [15] derived a model for gas flow through a straight capillary tube with a correction of the slip boundary condition. He then introduced the so-called apparent gas permeability for flow through an idealized porous medium that consists of a bundle of capillary tubes with the same diameter. The apparent permeability can be written as a correction factor multiplied by the intrinsic permeability, $k$, as follows:

$$
k_{a}=\left(1+\frac{d}{\bar{p}}\right) k
$$

where $d$ is a constant and $\bar{p}$ is the reciprocal mean gas pressure.

Civan [19] used the BK tube model and derived a unified apparent permeability model for an idealized porous medium blueconsisting of a bundle of tubes. Such model has a similar form as Equation (7) by replacing $(1+d / \bar{p})$ with $f(\mathrm{Kn})$

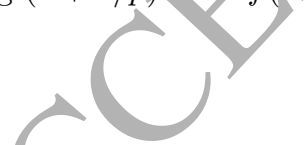

$$
k_{a}=f(\mathrm{Kn}) k,
$$

where again $k$ is the intrinsic permeability of the porous medium, which was given by the Kozeny-Carman model in Civan [19].

We note that the bundle-of-nano-tubes model from Civan [19] is a simplified apparent permeability model for a nano-porous medium and can overestimate the apparent permeability as reported by Landry et al. [27]. More sophisticated models may be developed by considering the complex pore structures observed in natural shale formations. 


\subsubsection{Adsorption/desorption and surface diffusion of adsorbed gas}

The nano-tubes, or nano-porous materials, lead to extremely small pores and thus large surface areas on which gas can be adsorbed. The adsorbed gas may also migrate along the pore wall, which is often considered as a diffusion process that is named surface diffusion [40,41]. The amount of adsorption depends on the pressure and temperature of the free gas, and is often modeled by an isotherm that relates the amount of adsorption to the pressure of the free gas at a constant temperature. We discuss the details of the models for adsorbed gas in the following section when we consider specific shale material constituents.

\subsection{Gas transport in shale}

Digital images of shale show strong heterogeneity of the different material constituents (e.g., Figure 1). In this section, based on the FIB-SEM images in Figure 1, we consider four material constituents: macro-pores, organic matter, clay minerals, and granular minerals, and we employ different models for each of them. Organic matter and clay both contain very small unresolved pores that are below the image resolution. In these sub-resolution pores, non-Darcy effects (i.e., slip flow and Knudsen diffusion), adsorption/desorption, and surface diffusion can play important roles. We consider these unresolved regions as nano-porous materials with connected sub-resolution pores and pore throats, and model the various gas transport mechanisms at the continuum scale. Here, we use the simple bundle-of-nano-tubes model for the unresolved nano-porous regions. The granular minerals have negligible porosity, allowing us to consider them as impermeable materials, i.e., zero porosity and zero permeability. The macro-pores are pores that are fully resolved in the image; these macro-pores may belong to organic matter, clay minerals, or granular minerals. They can also bridge these three constituents. We model gas transport in macro-pores using the Stokes equation. This is justified based on the assumption that the macro-pores have large sizes such that that the flow is either in the continuum flow regime, or the slip flow regime, where the Navier-Stokes equations (with velocity slip conditions) are applicable.

\subsubsection{Gas transport in organic matter}

We consider the organic matter with sub-resolution pores as a nano-porous medium, and derive an apparent permeability model based on section 2.1. In addition, we consider gas adsorption and surface diffusion.

\section{Model for the Apparent Permeability}

The organic matter has mostly circular pores and pore throats (see for example the HIM images in Wu et al. [13]). For the apparent permeability, we use the correction function from the bundle-of-nano-tubes model derived from the BK model for radial tubes. Thus, we have

$$
f(\mathrm{Kn})=\left(1+\alpha_{\mathrm{om}} \mathrm{Kn}\right)\left(1+\frac{4 \mathrm{Kn}}{1+\mathrm{Kn}}\right)
$$


where $\alpha_{\mathrm{om}}=a_{0, \mathrm{om}} \frac{2}{\pi} \tan ^{-1}\left(\alpha_{1, \mathrm{om}} \mathrm{Kn}^{\beta_{\mathrm{om}}}\right)$ with $a_{0, \mathrm{om}}=\frac{64}{15 \pi}, \alpha_{1, \mathrm{om}}=4.0, \beta_{\mathrm{om}}=0.4$. The subscript 'om' denotes organic matter.

Then, using the correction function, flow of free gas in the organic matter can be described by the Darcy-type equation with an apparent permeability $k_{a, \mathrm{om}}$ as

$$
\mathbf{u}_{f}=-\frac{k_{a, \mathrm{om}}}{\mu_{f}} \nabla p_{f}=-\frac{\left(1+\alpha_{\mathrm{om}} \mathrm{Kn}\right)\left(1+\frac{4 \mathrm{Kn}}{1+\mathrm{Kn}}\right) k_{\mathrm{om}}}{\mu_{f}} \nabla p_{f} .
$$

Since the sub-resolution pores in the organic matter are not resolved in the image, we need to make approximations for the porosity, permeability, and the average pore radius. Some of the information can be estimated from higher resolution TEM, or HIM, images of the organic matter and the pore size distribution (PSD) measured from nitrogen adsorption. We obtain the Kn number based on the average radius and compute the intrinsic permeability $k_{\mathrm{om}}$ using the Kozeny-Carman model using the estimated porosity.

Experiments have shown that the Langmuir isotherm fits methane adsorption in organic-rich shale and isolated kerogen reasonably well $[42,43]$. As a result, we use the Langmuir isotherm to model methane adsorption in the organic matter.

$$
n_{\mathrm{ad}}=n_{\mathrm{ad}}^{\max } \frac{K p_{f}}{1+K p_{f}}
$$

where $n_{\text {ad }}$ is the amount of adsorbed gas (mass per unit volume of porous material), $n_{\mathrm{ad}}^{\max }$ is the maximum adsorption, $K$ is the Langmuir coefficient, which is the inverse of the pressure at which half of the adsorption sites are occupied.

\section{Surface diffusion}

The adsorbed methane can migrate along the pore wall through a process referred to as surface diffusion $[40,41]$. The volumetric flux of the adsorbed gas in organic matter due to surface diffusion can be written as

$$
\mathbf{u}_{\mathrm{ad}}=-\frac{1}{\rho_{\mathrm{ad}}} D_{s} \nabla n_{\mathrm{ad}}=-\frac{1}{\rho_{\mathrm{ad}}} D_{s} \nabla\left(n_{\mathrm{ad}}^{\max } \frac{K p_{f}}{1+K p_{f}}\right),
$$

where $\mathbf{u}_{\mathrm{ad}}$ is the volumetric flow rate per unit area, $\rho_{\mathrm{ad}}$ is the density of the adsorbed gas, $D_{s}$ is the surface diffusivity. Note that we assume the diffusion is isotropic and thus $D_{s}$ is a scalar.

\subsubsection{Gas transport in clay}

Here, we discuss the gas flow model in clay, which is also modeled as a nano-porous medium with subresolution pores. Gas adsorption in clay may be negligible due to the presence of water in natural shale formations [see 44, 43]. Thus, we do not consider gas adsorption effects in clay. When adsorption is considered in clay, swelling mechanisms may need to be modeled [e.g., 45]. Unlike the circular pores in organic matter, 
clay has slit-like pores; as a result, we use the correction function from the bundle-of-nano-tubes model derived from the BK model for rectangular tubes. Namely,

$$
f(\mathrm{Kn})=\left(1+\alpha_{\mathrm{c}} \mathrm{Kn}\right)\left(1+\frac{6 \mathrm{Kn}}{1+\mathrm{Kn}}\right) .
$$

where $\alpha_{c}$ follows the same empirical relation as in the radial tube case, with different coefficients $\alpha_{0, \mathrm{c}}, \alpha_{1, \mathrm{c}}$, and $\beta_{\mathrm{c}}$ that change with the aspect ratio $(\mathrm{AR}=w / h)$ of the rectangular tube. The subscript ' $\mathrm{c}$ ' represents clay.

Then, the volumetric flux of free gas in clay becomes

$$
\mathbf{u}_{f}=-\frac{k_{a, \mathrm{c}}}{\mu_{f}} \nabla p_{f}=-\frac{\left(1+\alpha_{\mathrm{c}} \mathrm{Kn}\right)\left(1+\frac{6 \mathrm{Kn}}{1+\mathrm{Kn}}\right) k_{\mathrm{c}}}{\mu_{f}} \nabla p_{f}
$$

where $k_{a, \mathrm{c}}$ and $k_{\mathrm{c}}$ are the apparent permeability and intrinsic permeability of clay, respectively.

Similar to the organic matter, we obtain Kn using the estimated average pore thickness in clay. The intrinsic permeability $k_{c}$ of clay is approximated with the Kozeny Carman model with estimated porosity.

\subsubsection{Gas transport in macro-pores}

We assume that the macro-pores have large sizes that the flow is either in the continuum flow regime, or the slip flow regime where the Navier-Stokes equations (with velocity slip conditions) are applicable. Shale is known to have very low permeability (from nanoDarcy to microDarcy); therefore, we neglect inertial effects and use the Stokes equation to model gas flow in the macro-pores. We use the following equation for compressible flow in the macropore (see Appendix $B$ for a scaling argument).

$$
0=-\nabla p_{f}+\rho_{f} \mathbf{g}+\nabla \cdot\left(\mu_{f} \nabla \mathbf{u}_{f}\right)+\frac{1}{3} \nabla\left(\mu_{f} \nabla \cdot \mathbf{u}_{f}\right) .
$$

By considering appropriate velocity slip conditions at the wall of the macro-pores, the Stokes equation is applicable for both the continuum flow regime and the slip flow regime.

\section{Micro-continuum modeling framework}

We cast the models for gas transport in the different material constituents (organic matter, clay minerals, macro-pores, and granular minerals) into a micro-continuum modeling framework [34]. The micro-continuum framework allows us to solve Stokes flow in the macro-pores and continuum-scale flow in the nanoporous organic matter and clay without explicit coupling at the boundaries between the different constituents. It has been shown that the single-momentum equation approach is a good approximation of the explicit coupling between Stokes and Darcy equations using the Beavers-Joseph condition [46, 47]. We present the mathematical model and the numerical algorithm in the following. 


\subsection{Mathematical model}

The micro-continuum modeling framework is based on the Darcy-Brinkman-Stokes equations [35], which have been recently used to model fluid flow in conventional rocks at the pore-scale, such as carbonate [48, 34].

We consider compressible flow using the equation of states from section 2.1.1. The mass balance equation for methane, including both the free and adsorbed phases, can be written as

$$
\frac{\partial\left(\phi \rho_{f}\right)}{\partial t}+\delta_{\mathrm{om}}\left(1-\frac{\rho_{f}}{\rho_{\mathrm{ad}}}\right) \frac{\partial n_{\mathrm{ad}}}{\partial t}+\nabla \cdot\left(\rho_{f} \mathbf{u}_{f}+\delta_{\mathrm{om}} \rho_{\mathrm{ad}} \mathbf{u}_{\mathrm{ad}}\right)=0
$$

where $\phi$ is the porosity, which is between 0 and 1 in the region with sub-resolution pores (i.e., organic matter and clay). The porosity is zero in granular minerals and unity in macro-pores; $\delta_{\mathrm{om}}$ is an indicator for the organic matter, $\delta_{\mathrm{om}}=1$ when it is in the organic matter, otherwise $\delta_{\mathrm{om}}=0$. We multiply the terms associated with adsorption by $\delta_{\text {om }}$ because we only consider adsorption/desorption in the organic matter. We note that the adsorbed gas occupies a fraction of the porosity that would otherwise be filled with free gas. The reduction of the porosity due to adsorption needs to be taken into account [42] by using the excess adsorption, $\left(1-\frac{\rho_{f}}{\rho_{\mathrm{ad}}}\right) n_{\mathrm{ad}}$, which is defined as the absolute adsorption $n_{\mathrm{ad}}$ subtracted by the mass of the free gas that is replaced by the adsorbed gas, $\frac{\rho_{f}}{\rho_{\text {ad }}} n_{\text {ad }}$.

For the momentum equation, we use the compressible Darcy-Brinkman-Stokes equation to link the models for gas transport in the organic matter, clay, and macro-pores/see Equation (17)). Equation (17) recovers Equation (15) in the macro-pores, and recovers the Darcy-type equation $\mathbf{u}_{f}=-\frac{k_{a}}{\mu_{f}} \nabla p_{f}$ in the nano-porous region with sub-resolution pores. We note that the permeability $k_{a}$ is not only a function of the pore structure, it also depends on the flow properties, e.g., Kn number, and follows Equations (10) and (14) in the organic matter and clay regions, respectively. We consider no-slip condition at the interface between the macro-pores and the granular minerals.

$$
0=-\nabla p_{f}+\rho_{f} \mathbf{g}+\frac{1}{\phi} \nabla \cdot\left(\mu_{f} \nabla \mathbf{u}_{f}\right)+\frac{1}{3 \phi} \nabla\left(\mu_{f} \nabla \cdot \mathbf{u}_{f}\right)-\mu_{f} k_{\mathrm{a}}^{-1} \mathbf{u}_{f}
$$

\subsection{Numerical algorithm}

We implement and solve the set of equations for the micro-continuum framework (Equations (16) and (17)) in OpenFOAM $\mathbb{R}$ based on the PIMPLE solver, which is a transient solver for the Navier-Stokes equations that merges the PISO (Pressure Implicit Splitting Operator) [49] and SIMPLE (Semi-Implicit Method of Pressure-Linked Equations) algorithms [50]. We present the details of the discretization and the PIMPLE algorithm to solve Equations (16) and (17) in Appendix C.

\section{Results and analysis}

Now, we use the micro-continuum pore-scale model to analyze how different physical transport mechanisms describe the overall transport in complex 3D models of shale based on high-resolution images of the shale pore structures and material constituents. We design two types of numerical experiments. One uses 


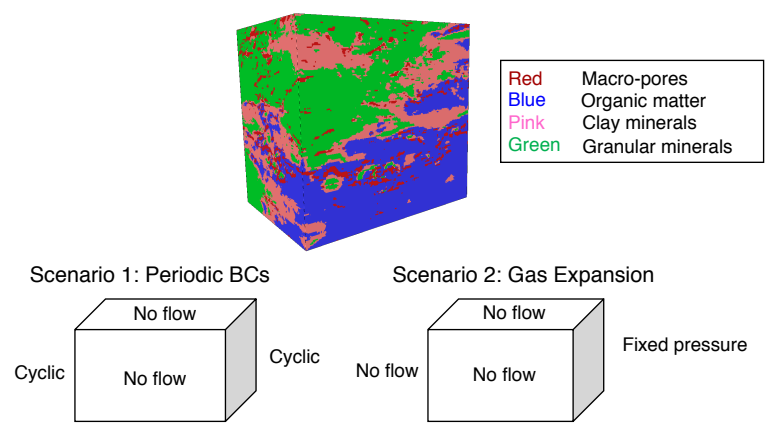

Figure 2: Sketches of two numerical experiments for simulating gas transport on a 3D FIB-SEM imagé. In both scenarios, left and/or right boundaries are open for flow and the other four sizes are no-flow boundaries. The first scenario considers periodic boundary conditions to compute an apparent permeability, and the second scenario only allows the right boundary to be open at a fixed pressure $\left(p_{f_{2}}\right)$ that is lower than the initial pressure in the sample $\left(p_{f_{1}}=p_{f_{2}}+\Delta p\right)$. The difference between the two pressures is small, $\Delta p \ll p_{f_{2}}$.

periodic boundary conditions, where we are interested in computing the overall flow capacity of the sample (i.e., the apparent permeability). The other setting uses Dirichlet boundary conditions to simulate the transient process of gas production. In both cases, the left and/or the right faces of the sample are open for flow and the other four sides of the sample are closed (see Figure 2). To analyze the importance of different physics or transport mechanisms, we consider four levels of model complexity:

(1) Darcy+Stokes (DS): gas flow in organic matter and clay is modeled with Darcy's law, and Stokes equation is used for flow in macro-pores;

(2) Darcy+Stokes+adsorption/desorption (DSA): adsorption/desorption is considered in organic matter in addition to the DS model;

(3) nonDarcy+Stokes+adsorption/desorption (NDSA): non-Darcy effects are included in both organic matter and clay, with everything else the same as the DSA model;

(4) nonDarcy+Stokes4adsorption/desorption+surface diffusion (full model): this refers to the NDSA model with effects due to surface diffusion of the adsorbed methane.

In the following subsections, we introduce the FIB-SEM images. Then we present the simulation results and analysis for the two numerical experiments and for all of the four models.

\subsection{FIB-SEM images used for the flow simulations}

We use a subset of the original image shown in Figure 1 for our pore-scale simulation. This is because using the original full image is computationally expensive and becomes prohibitive for our detailed analysis. The sub-image is cut with each dimension being about half of the original full image. The size of the subimage is $L_{x}=3.56 \mu \mathrm{m}, L_{y}=2.50 \mu \mathrm{m}, L_{z}=3.36 \mu \mathrm{m}$, and the voxel size is the same as the original full image ( $\Delta x=\Delta y=10 \mathrm{~nm}, \Delta z=20 \mathrm{~nm}$ ). Figure 3 shows distributions of the four material constituents in the sub-image. We also identify the two largest connected macro-pore clusters in Figure 3(f), which shows that there is a macro-pore network (the largest cluster) connecting the left face to the right face of the image. 
(a)

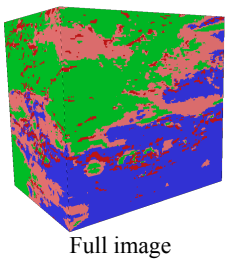

(c)
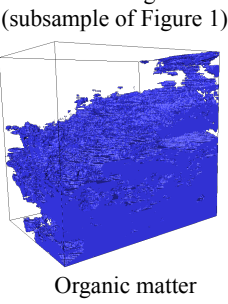

(e)

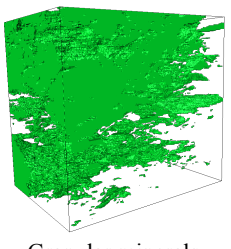

(b)

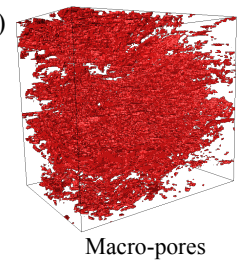

(d)

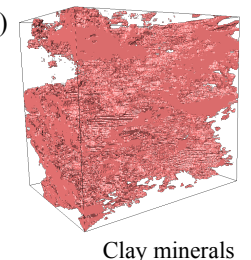

(f)

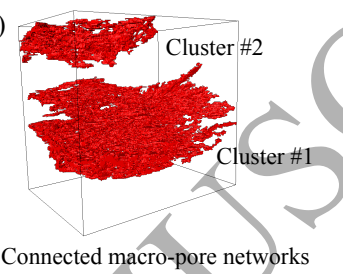

Figure 3: A subsample of the full image in Figure 1. Size of the image: $3.56 \mu \mathrm{m} \times 2.50 \mu \mathrm{m} \times 3.36 \mu \mathrm{m}$. (a) shows the overall image, and (b)-(e) show the distributions of the four material constituents: red - macro-pores, blue - organic matter, pink clay minerals, green - granular minerals; (f) shows the two largest connected macro-pore networks.

We will see later that this pore structure has a significant impact on the results of the simulations. The 3D images were acquired in a Dual Beam FIB-SEM (Nova NanoLab 600i, FEI, Hillsboro, United States) and they were aligned and sheared as a series of block face images before segmentation. A band-pass filter, non-local means filter, sober filter and a top-hat filter were applied for the minerals, organic matter and pores segmentation. More details about the shale core sample, the acquisition procedures of the image, the image processing and other measurements can be found in [12]

We discretize the image with the finite-volume scheme and solve Equations (16) and (17) subject to the boundary conditions of the two numerical experiments. The numerical grid cells have a one-to-one correspondence with the digital voxels of the image, which leads to 14, 952, 000 grid cells. Each voxel belongs to one of the four material constituents, and is given a porosity. Voxels with $\phi=1$ belong to macro-pores, $\phi=0$ belong to granular minerals, and $\phi \in(0,1)$ represents organic matter and clay. $\delta_{\text {om }}=1$ when a voxel sits in organic matter, otherwise, $\delta_{\mathrm{om}}=0$.

\subsection{D simulations with periodic BCs: computing apparent permeability}

In this section, we present the results and analysis of the first numerical experiment, which includes two parts. The first part computes and analyzes the apparent permeability of organic matter and clay, and the second part considers the apparent permeability of the $3 \mathrm{D}$ image.

Here, we introduce the parameters we use for the simulation (see Table 1). The organic matter and the 


\begin{tabular}{lc}
\hline Parameter & Value \\
porosity of organic matter $\left(\phi_{\mathrm{om}}\right)$ & 0.1 \\
porosity of clay $\left(\phi_{\mathrm{c}}\right)$ & 0.1 \\
average pore size in organic matter & $5 \mathrm{~nm}$ \\
average pore size in clay & $5 \mathrm{~nm}$ \\
Langmuir coefficient $(K)$ & $4 \times 10^{-8} \mathrm{~Pa}^{-1}$ \\
maximum adsorption $\left(n_{\mathrm{ad}}^{\max }\right)$ & $44.8 \mathrm{~kg} / \mathrm{m}^{3}$ \\
surface diffusion coefficient $\left(D_{s}\right)$ & see Table 2 \\
density of adsorbed gas $\left(\rho_{\mathrm{ad}}\right)$ & $400 \mathrm{~kg} / \mathrm{m}^{3}$ \\
temperature & $400 \mathrm{~K}$ \\
\hline
\end{tabular}

Table 1: Parameters used for the simulations. Note that organic matter is assumed to have radial pores and the average pore size is the average pore radius; clay is assumed to have slit-like pores and the average pore size refers to the average pore thickness.

\begin{tabular}{lcccccccc}
\hline$p_{f}[\mathrm{MPa}]$ & 1 & 5 & 10 & 20 & 30 & 40 & 50 \\
$D_{s}\left[10^{-8} \mathrm{~m}^{2} / \mathrm{s}\right]$ & $8.32 \times 10^{-1}$ & $9.52 \times 10^{-1}$ & 1.14 & 1.44 & 1.77 & 2.10 & 2.46 \\
\hline
\end{tabular}

Table 2: Surface diffusion coefficient as function of gas pressure.

clay are assumed to be homogeneous with a porosity of 0.1 . The organic matter has an average pore radius of $5 \mathrm{~nm}$ and the clay slit-pores have an average height of $5 \mathrm{~nm}$. The equation of state and the viscosity equation of methane give the density and viscosity range from $p=1 \mathrm{MPa}$ to $p=50 \mathrm{MPa}$ (see Figure 4 ). Details of the equation of state and viscosity equation are shown in Appendix A. We consider an isothermal system with a temperature of $400 \mathrm{~K}$. As we can see, density of methane changes drastically from $p=1 \mathrm{MPa}$ to $p=50 \mathrm{MPa}$, while the viscosity has much less variation. The critical pressure of methane $p_{\text {critical }}=4.599 \mathrm{MPa}$ and the critical temperature $T_{\text {critical }}=190.564 \mathrm{~K}$. At $T=400 \mathrm{~K}$, methane enters the supercritical phase when pressure is higher than the critical pressure $p_{\text {critical }}=4.599 \mathrm{MPa}$. The Langmuir coefficient, maximum adsorption, and density of adsorbed gas are typical parameters for isolated organic matter obtained from Rexer et a1. [43]. The surface diffusion coefficient $\left(D_{s}\right)$ is a function of the amount of adsorption, and therefore depends on pressure. Here we take typical values from Medved̆ and Černỳ [41] as shown in Table 2. $D_{s}=D_{s 0} D_{s} / D_{s 0}$, where $D_{s 0}=8.00 \times 10^{-9} \mathrm{~m}^{2} / \mathrm{s}$, and the ratio $D_{s} / D_{s 0}$ is taken from the Langmuir/HIO curve in the Figure 4 of Medved and Černỳ [41]. We note that these parameters are typical parameters for surface diffusion in activated carbon obtained from the literature, though different parameters can be used, we do not expect that they change the qualitative behavior of the simulations. 

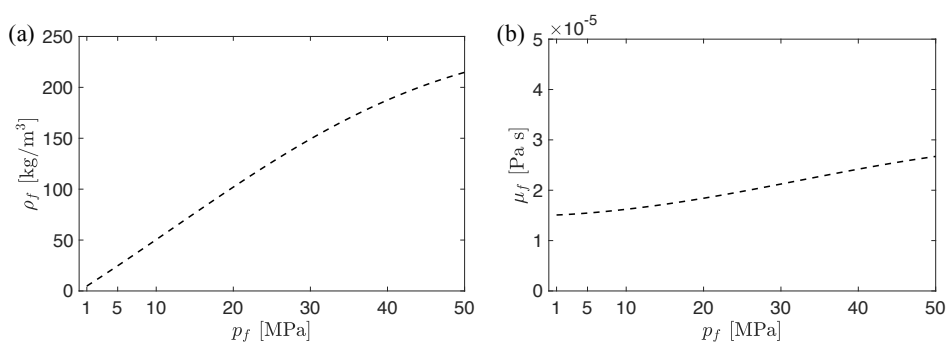

Figure 4: Variations of density (a) and viscosity (b) of methane with gas pressure,

\subsubsection{Apparent permeability of clay and organic matter}

The apparent permeability of organic matter and clay can be computed analytically from the models presented in section 2.2 .

The apparent permeability of clay can be obtained from Equation (14) as:

$$
\left.k_{a, \mathrm{c}}=\left(1+\alpha_{\mathrm{c}} \mathrm{Kn}\right)\left(1+\frac{6 \mathrm{Kn}}{1+\mathrm{Kn}}\right) k_{\mathrm{c}}\right)
$$

where $\alpha_{\mathrm{c}}=\alpha_{0, \mathrm{c}} \frac{2}{\pi} \tan ^{-1}\left(\alpha_{1, \mathrm{c}} \mathrm{Kn}^{\beta_{\mathrm{c}}}\right)$ with $\alpha_{0, \mathrm{c}}=1.5272, \alpha_{1, \mathrm{c}}=2.5, \beta_{\mathrm{c}}=0.5$. The coefficients $\alpha_{0, \mathrm{c}}, \alpha_{1, \mathrm{c}}$, and $\beta_{\mathrm{c}}$ are parameters for a rectangular tube with an aspect ratio of $4(w / h=4)$ [17]. Here, we assume the clay pores have an averaged aspect ratio of 4 . Different aspect ratio leads to different coefficients, but only with a slight modification to $\alpha_{c}$. The intrinsic permeability of clay $k_{\mathrm{c}}$ is obtained using the Kozeny-Carman model with an average porosity of 0.1 . We recognize that the Kozeny-Carman model may not be applicable to the clay with slit-like pores, the idea here is to assign a reasonable intrinsic permeability to the clay minerals. A better intrinsic permeability may be assigned if higher resolution images of the nano-porous clay region are available.

The apparent permeability of the organic matter is more complex, since it involves the flux of the adsorbed gas (surface diffusion). To include surface diffusion, we convert the mass flux of the adsorbed gas into volumetric flux of free gas and combine it with the free gas flux to obtain the apparent permeability

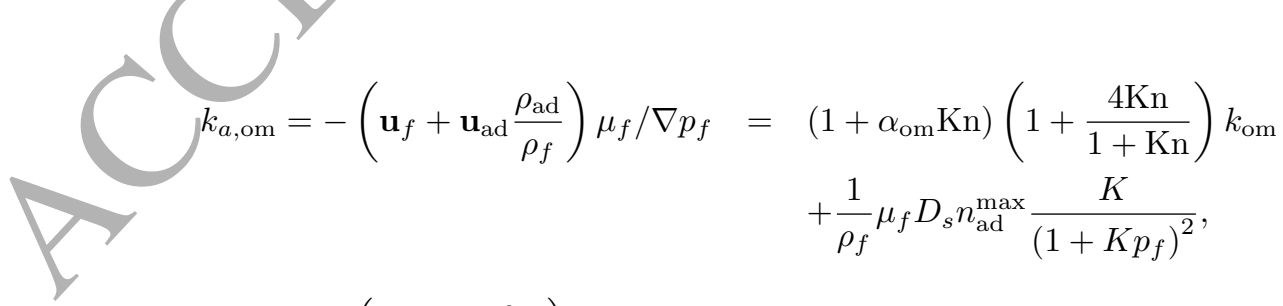

where $\alpha_{\mathrm{om}}=\alpha_{0, \mathrm{om}} \frac{2}{\pi} \tan ^{-1}\left(\alpha_{1, \mathrm{om}} \mathrm{Kn}^{\beta_{\mathrm{om}}}\right)$ with $\alpha_{0, \mathrm{om}}=1.358, \alpha_{1, \mathrm{om}}=4.0, \beta_{\mathrm{om}}=0.4$ [17]. Similar to clay, the intrinsic permeability of organic matter $k_{\mathrm{om}}$ is obtained with the Kozeny-Carman model with an average porosity of 0.1 . Here we compute the apparent permeability of organic matter both with and without surface diffusion.

From Equations (18) and (19), we plot the apparent permeability for clay and organic matter in Figure 


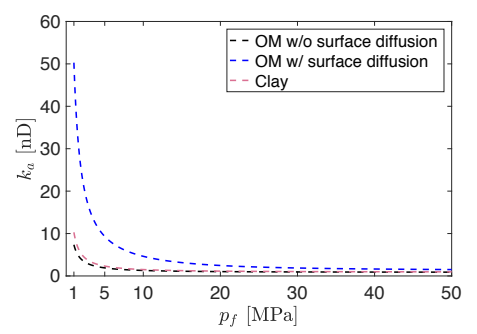

Figure 5: Apparent permeability of organic matter and clay as a function of gas pressure.

5 as a function of gas pressure. The intrinsic permeabilities $k_{\mathrm{c}}$ and $k_{\mathrm{om}}$ are both $0.69 \mathrm{nD}$ ( $\mathrm{nD}$ denotes nano Darcy, which is $10^{-21} \mathrm{~m}^{2}$ ). The apparent permeabilities are a strong function of pressure that have arge values at $p_{f}=1 \mathrm{MPa}$ and then quickly decrease for higher pressures. At $p_{f}=1 \mathrm{MPa}, k_{\mathrm{c}}=10.29 \mathrm{nD}, k_{\mathrm{om}}=$ $7.35 \mathrm{nD}$ without surface diffusion, and $k_{\mathrm{om}}=50.28 \mathrm{nD}$ when surface diffusion is included; at $p_{f}=50 \mathrm{MPa}$, $k_{\mathrm{c}}=0.97 \mathrm{nD}, k_{\mathrm{om}}=0.90 \mathrm{nD}$ without surface diffusion, and $k_{\mathrm{om}}=1.51 \mathrm{nD}$ when surface diffusion is included. We can see that surface diffusion significantly increases the apparent permeability of the organic matter at low pressure ( $\sim 7$ times of the apparent permeability without surface diffusion at $\left.p_{f}=1 \mathrm{MPa}\right)$; surface diffusion effects become less important for high pressures $(\sim 1.7$ times of the apparent permeability without surface diffusion at $p_{f}=50 \mathrm{MPa}$ ).

\subsubsection{Apparent permeability of the 3D sample}

Now we take the image (Figure 3) and compute the apparent permeability of the entire sample using periodic boundary conditions shown in Figure 2. We include a body force in the momentum equation to impose a pressure gradient in $x$ direction, and then solve Equations (16) and (17) subject to the periodic boundary conditions in $x$ direction until it reaches steady state. The pressure gradient we assign is $\partial p_{f} / \partial x=$ $10^{5} \mathrm{~Pa} / \mathrm{m}$. We use $q$ to denote the volumetric flow rate through the $y-z$ cross-section of the sample at steady state, which can be obtained as

$$
q=\int u_{x} \mathrm{dydz}
$$

Then, the apparent permeability of the sample in $x$ direction can be defined as

$$
k_{a, \text { sample }, x}=q /\left[\left(L_{y} L_{z}\right) \mu_{f} \frac{\partial p_{f}}{\partial x}\right] .
$$

We assign a reference pressure to the domain to compute an apparent permeability for a given pressure. The reference pressure is set at the right boundary, and the maximum difference of the pressure in the domain relative to the reference pressure is negligibly small, $L_{x} \partial p_{f} / \partial x=0.356 \mathrm{~Pa}$. We compute the apparent permeability for reference pressures $p_{f}=1,5,10,20,30,40,50 \mathrm{MPa}$, for all of the four models (DS, DSA, NDSA, and full model). Note that $q=q_{f}$ for the first three models (DS, DSA, NDSA) where there is only free gas flux, while $q=q_{f}+q_{\text {ad }} \frac{\rho_{\text {ad }}}{\rho_{f}}$ for the full model where surface diffusion is included. 


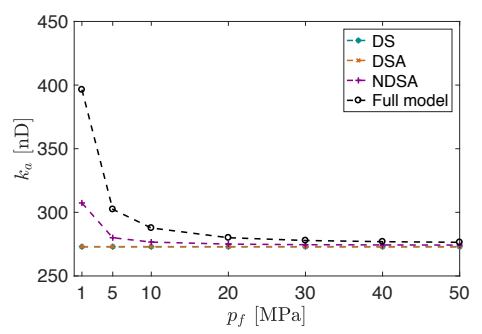

Figure 6: Apparent permeability of the 3D image in $x$ direction computed from the four models (DS, DSA, NDSA, and full model) using periodic BCs for gas pressures $p_{f}=1,5,10,20,30,40,50 \mathrm{MPa}$.

We summarize the computed apparent permeabilities in Figure 6, from which we can make several observations. First, because of the connected macro-pore network (Figure $3(\mathrm{f})$ ), the permeability of the sample from the DS model $\left(k_{\mathrm{DS}}=272.98 \mathrm{nD}\right)$ is much larger than the intrinsic permeability of clay and organic matter $(0.69 \mathrm{nD})$. Second, $k_{\mathrm{DSA}}=k_{\mathrm{DS}}$ since adsorption does not change permeability at steady state. Third, apparent permeabilities from the NDSA model and full model are higher, especially at low pressure, $k_{\mathrm{NDSA}}=307.38 \mathrm{nD}$ and $k_{\text {full model }}=396.41 \mathrm{nD}$ at $p_{f}=1 \mathrm{MPa}$. However, the increase relative to the DS permeability (less than $\sim 1.5$ times of $k_{\mathrm{DS}}$ ) is much smaller compared to the increase of the apparent permeability of clay and organic matter relative to their intrinsic permeability (see Figure 5). This again shows that the connected macro-pore network completely dominates the permeability of the sample, which undermines the enhancement of flow capacity from non-Darcy effects and surface diffusion.

\subsection{D simulations of gas expansion: simulating gas production}

In this section, we present the second numerical experiment where we model gas expansion to simulate gas production. Before we show the simulation results, we introduce a simplified one-dimensional (1D) model for our system, which will be used to interpret the results of the 3D simulations.

\subsubsection{One-dimensional model for gas expansion}

Because only the right boundary of the domain is open for flow in our gas expansion system, it may be reasonable to approximate gas production as a 1D problem. This 1D model is similar to the flow model for pressure-pulse decay method $[51,52,53]$. For simplicity, we assume homogeneous properties of the domain. The porosity of the sample is $\bar{\phi}$ and the volume fraction of organic matter is $\bar{\gamma}_{\text {om }}$. The sample has an apparent permeability $\bar{k}_{a}$ when surface diffusion is not included. Here we consider surface diffusion separately. Then, the mass balance equation of the 1D model can be written as

$$
\frac{\partial\left(\bar{\phi} \rho_{f}\right)}{\partial t}+\bar{\gamma}_{\mathrm{om}} \frac{\partial n_{\mathrm{ad}}}{\partial t}\left(1-\frac{\rho_{f}}{\rho_{\mathrm{ad}}}\right)+\frac{\partial\left(\rho_{f} u_{f, x}+\bar{\gamma}_{\mathrm{om}} \rho_{\mathrm{ad}} u_{\mathrm{ad}, x}\right)}{\partial x}=0 .
$$

The pressure boundary condition only introduces a small pressure perturbation to the domain $(\Delta p=$ $\left.p_{f_{1}}-p_{f_{2}} \ll p_{f_{2}}\right)$, so that Equation (22) can be considered as a linear equation. Rearranging Equation (22), 
we obtain a linear 1D diffusion equation

$$
\frac{\partial p_{f}}{\partial t}=\hat{k} \frac{\partial^{2} p_{f}}{\partial x^{2}}
$$

410

where

$$
\hat{k}=\left[\rho_{f} \frac{\bar{k}_{a}}{\mu_{f}}+\bar{\gamma}_{\mathrm{om}} D_{s} n_{\mathrm{ad}}^{\max } \frac{K}{\left(1+K p_{f}\right)^{2}}\right] /\left[\bar{\phi} \frac{\partial \rho_{f}}{\partial p_{f}}+\bar{\gamma}_{\mathrm{om}}\left(1-\frac{\rho_{f}}{\rho_{\mathrm{ad}}}\right) n_{\mathrm{ad}}^{\max } \frac{K}{\left(1+K p_{f}\right)^{2}}\right]
$$

The initial and boundary conditions are

$$
\begin{gathered}
p_{f}(x, t=0)=p_{f_{1}}, \\
\frac{\partial p_{f}}{\partial x}(x=0, t)=0, \\
p_{f}\left(x=L_{x}, t\right)=p_{f_{2}} .
\end{gathered}
$$

Substitution of the dimensionless variables into Equation (23) gives

$$
\frac{\partial P_{f}}{\partial T}=\frac{\hat{k}}{\hat{k}_{\mathrm{DS}}} \frac{\partial^{2} P_{f}}{\partial X^{2}}
$$

The corresponding dimensionless boundary conditions become

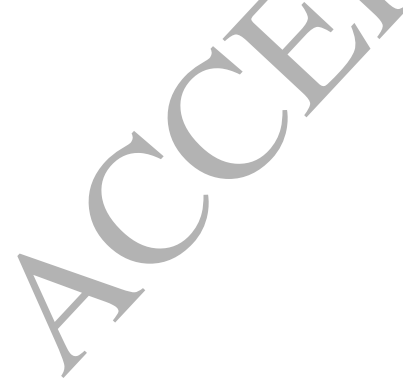

$$
\begin{gathered}
P_{f}(X, T=0)=1, \\
\frac{\partial P_{f}}{\partial X}(X=0, T)=0, \\
P_{f}(X=1, T)=0 .
\end{gathered}
$$

For a small pressure perturbation at the right boundary, $\frac{\hat{k}}{\hat{k}_{\mathrm{DS}}}$ can be considered as a constant, then

Equation (29) subject to the initial and boundary conditions (30)-(32) can be solved analytically

$$
P_{f}=\sum_{n=0}^{\infty} \frac{2}{(n+1 / 2) \pi} \sin [(n+1 / 2) \pi(1-X)] e^{-(n+1 / 2)^{2} \pi^{2} \frac{\hat{k}}{\hat{k}_{\mathrm{DS}}} T} .
$$


Approximating $P_{f}$ using the first term of the series solution (33) gives

$$
P_{f}=\frac{4}{\pi} \sin \left(\frac{1}{2} \pi(1-X)\right) e^{-\frac{1}{4} \pi^{2} \frac{\hat{k}}{\hat{k}_{\mathrm{DS}}} T} .
$$

From the pressure solution Equation (34), we can derive the expression for the outlet mass flow rate (production rate) at the right boundary. We nondimensionalize the outlet mass flow rate by $\dot{m}_{\mathrm{DS}}=$ $-\rho_{f} \frac{k_{\mathrm{DS}}}{\mu_{f}} \frac{p_{f_{2}}-p_{f_{1}}}{L_{x}}$, the characteristic outlet mass flow rate from the DS model, and obtain the dimensionless outlet mass flow rate (not including surface diffusion) as

$$
\dot{M}=\dot{m} / \dot{m}_{\mathrm{DS}}=-\left.\frac{\bar{k}_{a}}{k_{\mathrm{DS}}} \frac{\partial P_{f}}{\partial X}\right|_{X=1}=2 \frac{\bar{k}_{a}}{k_{\mathrm{DS}}} e^{-\frac{1}{4} \pi^{2} \frac{\hat{k}}{\hat{k}_{\mathrm{DS}}} T}
$$

When surface diffusion is included, the dimensionless outlet mass flow rate becomes

$$
\begin{aligned}
\dot{M} & =\dot{m} / \dot{m}_{c} \\
& =\frac{\left.\left[\rho_{f} u_{f, x}+\rho_{\mathrm{ad}} u_{\mathrm{ad}, x}\right]\right|_{X=1}}{\dot{m}_{c}} \\
& =2\left[\frac{\bar{k}_{a}}{k_{\mathrm{DS}}}+\bar{\gamma}_{\mathrm{om}} D_{s} n_{\mathrm{ad}}^{\max } \frac{K \mu_{f}}{\rho_{f} k_{\mathrm{DS}}\left(1+K p_{f}\right)^{2}}\right] e^{-\frac{1}{4} \pi^{2} \frac{\hat{k}}{\hat{k}_{\mathrm{DS}}} T} .
\end{aligned}
$$

Equations (35) and (36) show that the dimensionless outlet mass flow rate declines exponentially with dimensionless time, $T \cdot \ln \dot{M} \sim T$ with the slope $-\frac{1}{4} \pi^{2} \frac{\hat{k}}{\hat{k}_{\mathrm{DS}}}$ representing the flow capacity of the shale sample. In the following section, we use the results and analysis of the $1 \mathrm{D}$ model to interpret the production decline results from the $3 \mathrm{D}$ simulations of gas production.

\subsubsection{Production decline}

We simulate gas production for $p_{f}=1 \mathrm{MPa}$ and $p_{f}=50 \mathrm{MPa}$, representing low and high pressures, respectively. Pressure $p_{f}$ refers to the gas pressure at the right boundary $p_{f_{2}}$. The difference between the initial pressure $\left(p_{f_{1}}\right)$ in the domain and the boundary pressure $\left(p_{f_{2}}\right)$ is kept as $\Delta p=100 \mathrm{~Pa} \ll p_{f}$. Integrating the mass flux across the right boundary, we obtain the outlet mass flow rate. We normalize the mass flow rate using the characteristic mass flow rate from the DS model, $\dot{m}_{\mathrm{DS}}$, introduced in section 4.3.1, and nondimensionalize the time scale using the characteristic time scale from the DS model, $T_{\mathrm{DS}}=L_{x}^{2} / \hat{k}_{\mathrm{DS}}$. Then, we plot the dimensionless mass flow rate with the dimensionless time on a semi-log scale, for both early time $(0<T<0.1)$ and late time $(0<T<10)$ (see Figure 7 ).

Now we analyze the $3 \mathrm{D}$ gas production decline results using the $1 \mathrm{D}$ model in section 4.3.1. We first look at the early time behavior. At $p_{f}=1 \mathrm{MPa}$, starting from $T \approx 0.05$, the production rates decline linearly in the semi-log scale and follow a similar slope in all of the four models (see Figure 7 (a)), despite their very different model complexities. This is primarily due to the macro-pore network in the 3D image which connects the left boundary to the right boundary of the image, as shown in Figure 3(f). The macro-pore network dominates gas production in early time, in which gas flow follows Stokes equation in any of the four 

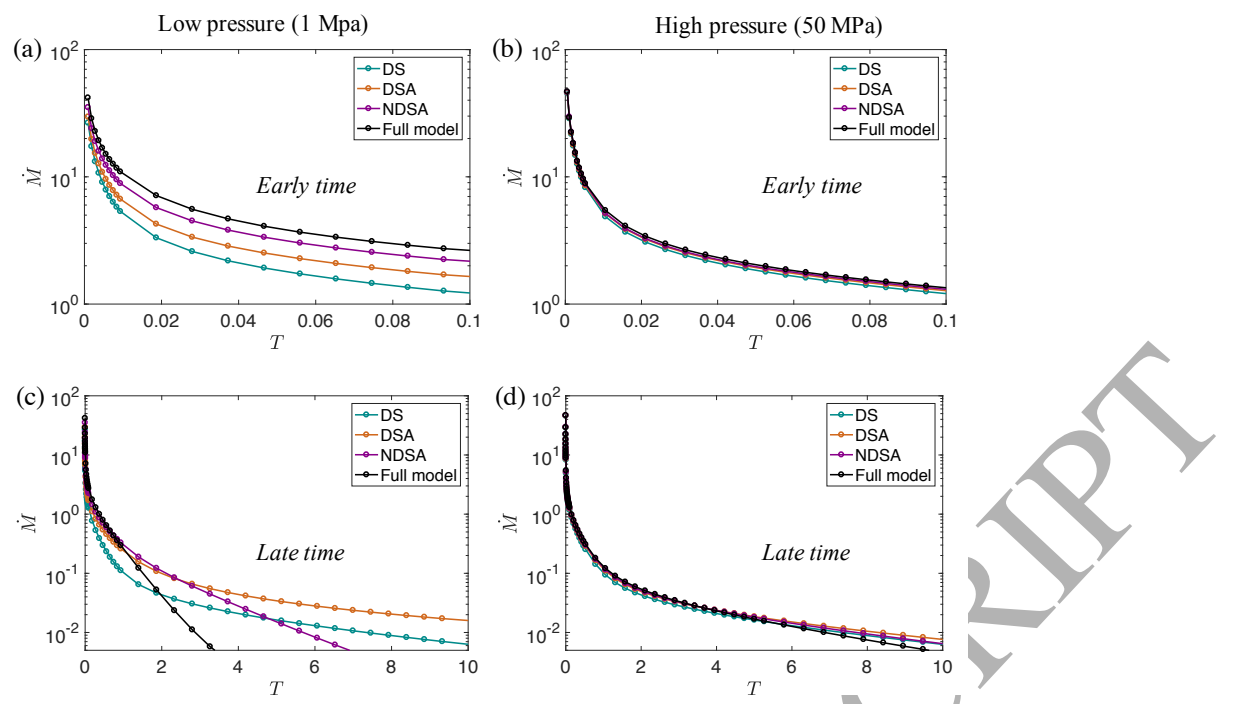

Figure 7: Simulation of gas production decline on the 3D image using the four models (DS, DSA, NDSA, and full model). (a) and (c) are early and late time decline for a low gas pressure $\left(p_{f}=1 \mathrm{MPa}\right)$, and (b) and (d) are early and late time decline for a high gas pressure $\left(p_{f}=50 \mathrm{MPa}\right)$.

models. Therefore, the four models share a similar slope in early time even though they have very different physics. We note that the gas production rates do not decline linearly in the very beginning up to $T \approx 0.05$. This is because the pressure signal at the right boundary has not reached the left boundary yet, and the system transits from a 'semi-infinite' like domain to a finite domain, which does not follow the exponential scaling derived from the $1 \mathrm{D}$ model in section 4.3.1. At $p_{f}=50 \mathrm{MPa}$, the production decline curves follow the same slope starting from $T \approx 0.05$ (see Figure $7(\mathrm{~b})$ ). In addition, the production rates from the four models almost overlap with each other. We note that the production decline curves from the DS model for $p_{f}=1 \mathrm{MPa}$ and $p_{f}=50 \mathrm{MPa}$ are almost identical, which shows that the impact from compressibility is eliminated through the nondimensionalization.

In late time, the production decline curves from low and high gas pressures are quite different (see Figure $7(\mathrm{~b})$ and $(\mathrm{d}))$. At $p_{f}=1 \mathrm{MPa}$, the production rates from the four models diverge and follow very different decline slopes. The DS and DSA models have similar slopes with the DSA slope being slightly smaller. The NDSA model has a larger slope, and the full model has the largest slope. This is because gas production in late time is dominated by gas transport in the sub-resolution nano-porous region. The DS and DSA models both use Darcy's law in the nano-porous region. The DSA model includes adsorption which gives a smaller diffusivity $\hat{k}$ in the $1 \mathrm{D}$ model, then a flatter slope $-\frac{1}{4} \pi^{2} \frac{\hat{k}}{\hat{k}_{\mathrm{DS}}}$. NDSA considers non-Darcy effects in both organic matter and clay, and therefore has a larger apparent permeability $\bar{k}_{a}$, which leads to a faster decline in production. In addition to the non-Darcy effects, the full model includes surface diffusion which further enhances gas transport in nano-porous region, as shown by the steepest decline curve. At $p_{f}=50 \mathrm{MPa}$, the production decline curves from the four models are almost indistinguishable. The full model has a slightly larger slope, while the DSA model has the smallest slope. This implies that the non-Darcy effects and 

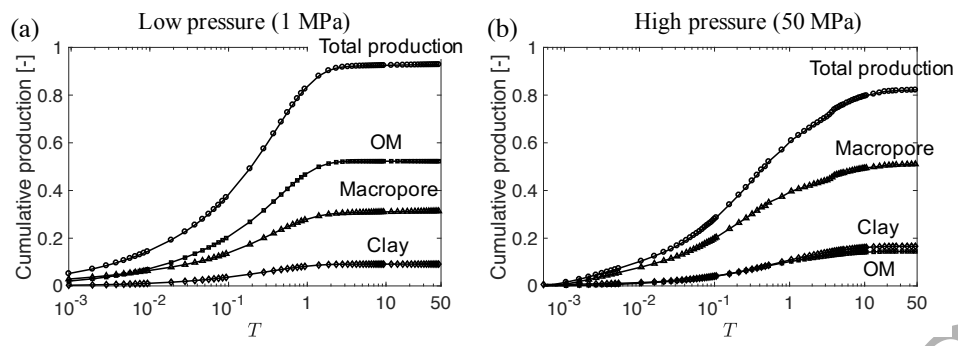

Figure 8: Simulation of cumulative gas production using the full model. (a) is for low pressure $\left(p_{f}=1 \mathrm{MPa}\right)$, and (b) is for high pressure $\left(p_{f}=50 \mathrm{MPa}\right)$. Total production as well as mass loss of gas in macro-pores, organic/matter, and clay are shown.

surface diffusion are negligible at high pressures, which is consistent with our observation in the periodic BCs numerical experiment (Figure 5). Again, we note that the production decline curves from the DS model for $p_{f}=1 \mathrm{MPa}$ and $p_{f}=50 \mathrm{MPa}$ are almost identical.

Finally, we point out that the transient analysis of gas production appears to be much more informative than the steady-state apparent permeability computed in section 4.2. The apparent permeability can be considered as a lumped average of the flow capacity of the sample over time, while the transient gas production process reveals the dependence of the flow capacity of the sample on time scales.

\subsubsection{Cumulative gas production}

The 3D simulations allow us to investigate the cumulative gas production. The simulation gives the gas density and pressure at each voxel of the image during gas production. Therefore, we can compute how much gas is produced from the entire domain, as well as, from each of the four material constituents (macro-pores, organic matter, clay, and granular minerals). We compute the theoretical total gas production of the sample as if the gas pressure drops from $p_{f_{1}}$ to $p_{f_{2}}$ at every voxel in the image (considering desorption), and use this theoretical total production to normalize the cumulative production rates. Figure 8 shows the results from the full model, where we plot the cumulative production from the entire sample and the loss of gas from each of the)four material constituents. The results for all of the four models are presented in Figures 9 and 10.

Analysis of the results in Figure 8 leads to two observations. First, the overall gas production is slightly less than unity, which means that there is gas in the domain that cannot be produced from the right boundary. This 'trapped' gas may stay in macro-pores, organic matter, or clay regions, isolated by the impermeable granular minerals not accessible from the right boundary. In fact, the second largest macro-pores cluster shown in Figure 3 is isolated by granular minerals, and the gas cannot be produced. Second, production from organic matter contributes the most at low pressure $\left(p_{f}=1 \mathrm{MPa}\right)$, while production from the macro-pores 

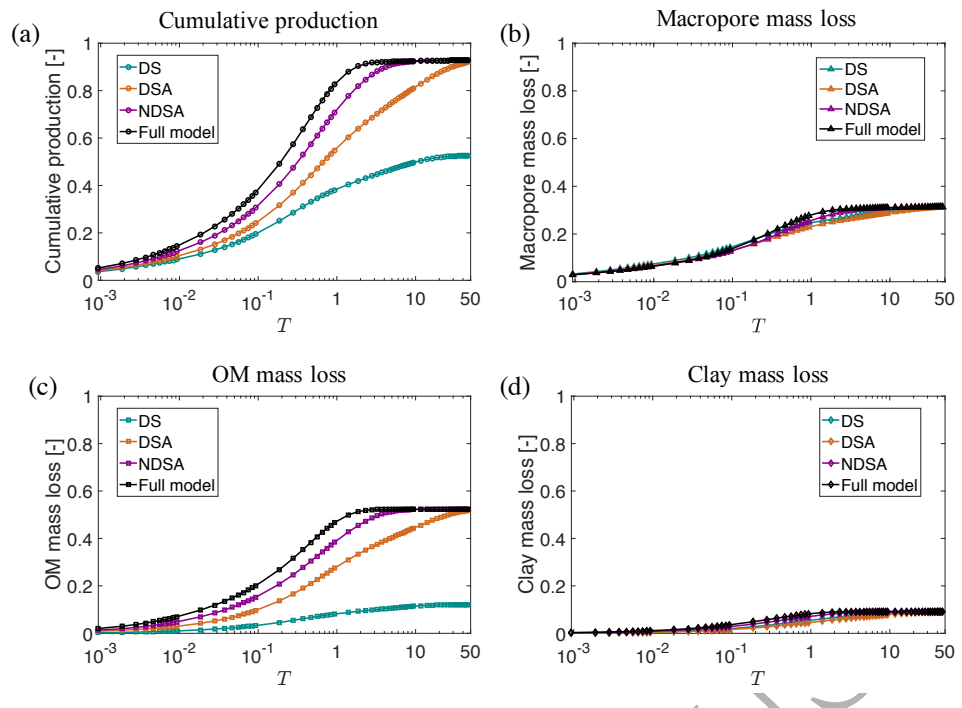

Figure 9: Simulation of cumulative gas production at low pressure ( $\left.p_{f}=1 \mathrm{MPa}\right)$ using the four models (DS, DSA, NDSA, and full model). (a) shows the total gas production, and (b)-(d) represent the mass loss in the sample from macro-pores, organic matter, and clay, over time.

(a)
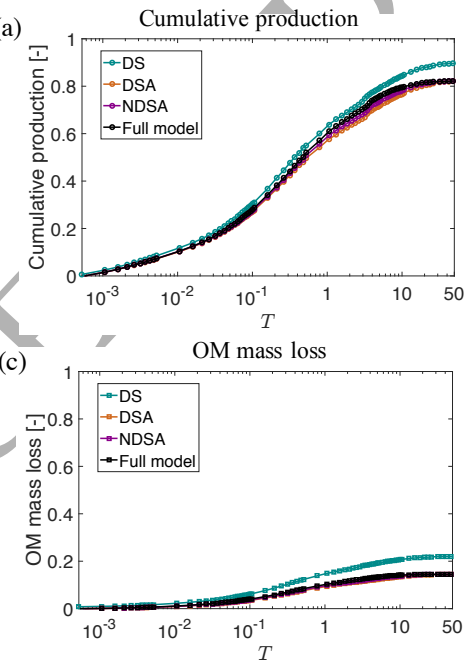

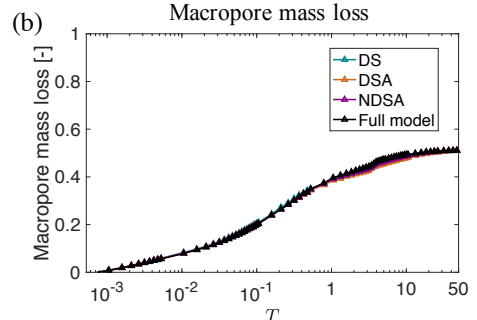

(d)

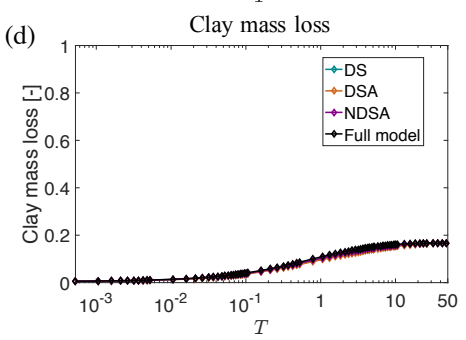

Figure 10: Simulation of cumulative gas production at high pressure $\left(p_{f}=50 \mathrm{MPa}\right)$ using the four models (DS, DSA, NDSA, and full model). (a) shows the total gas production, and (b)-(d) represent the mass loss in the sample from macro-pores, organic matter, and clay, over time. 


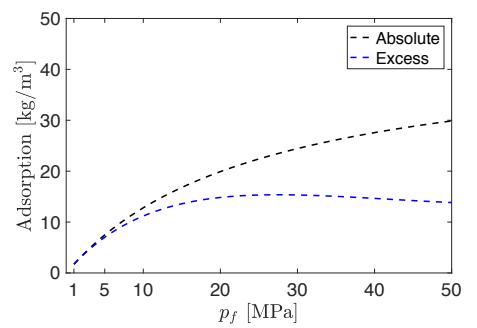

Figure 11: Absolute (black dashed line) and excess (blue dashed line) adsorbed mass per cubic meter of organic matter as a function of gas pressure for methane using typical parameters for isolated organic matter from organic-rich shale (Rexer et al. $[43])$.

dominates at high pressure $\left(p_{f}=50 \mathrm{MPa}\right)$. This is because the density contrast between the free-gas and the adsorbed gas is large at low pressure $\left(\rho_{\text {ad }}=400 \mathrm{~kg} / \mathrm{m}^{3}\right.$ and $\rho_{f}=4.84 \mathrm{~kg} / \mathrm{m}^{3}$ at $\left.p_{f}=1 \mathrm{MPa}\right)$, where production from desorbed gas can be significant. The contribution from desorption is less at high pressure as the density contrast is much smaller $\left(\rho_{\text {ad }}=400 \mathrm{~kg} / \mathrm{m}^{3}\right.$ and $\rho_{f}=214.74 \mathrm{~kg} / \mathrm{m}^{3}$ at $\left.p_{f}=50 \mathrm{MPa}\right)$.

Among the four models, DSA, NDSA, and the full model have the same total production for both low and high pressures. The total production from the DS model is less for low pressure, but more for high pressure (see Figures 9 and 10). This is because the excess adsorption changes from monotonically increasing at $p_{f}=1 \mathrm{MPa}$ to monotonically decreasing at $p_{f}=50 \mathrm{MPa}$, as shown in Figure 11 . Therefore, compared to the case with no adsorption/desorption (i.e., the DS model), adsorption/desorption leads to more production at low pressure, while less production at high pressure. At low pressure (Figure 9), we observe that as additional physical mechanisms are included, gas production is accelerated. Production from organic matter has the largest acceleration. However acceleration of gas production becomes negligible at high pressure as indicated in Figure 10, where cumulative production curves from the four models are almost identical. This confirms that the non-Darey effects and surface diffusion become negligible for high gas pressures.

The 3D simulations also allow us to visualize the gas production process at different simulation times. In Figure 12, we take the low pressure case $p_{f}=1 \mathrm{MPa}$ from the full model as an example to visualize gas production in 3D. We present the evolution of remaining producible gas over dimensionless time $(T=$ $0,0.01,0.1,1,50)$ during production. The producible gas is defined as the amount of gas that should be produced at each image voxel if gas pressure drops from $p_{f_{1}}$ to $p_{f_{2}}$. At $T=0$, the macro-pores have the highest producible gas density (mass of producible gas per voxel) indicated by the red colour. The green colour represents the producible gas density in the organic matter, while light blue indicates the producible gas in clay. The 3D visualization confirms our observations in Figure 7 that gas production is limited by the macro-pore network before $T=0.1$, while the nano-porous organic matter and clay become dominant in late time after $T=1$. Also, from $T=0$ to $T=50$, we can see that there is gas in domain (e.g., the red region at $T=50$ ) that is not accessible from the right boundary and cannot be produced. 


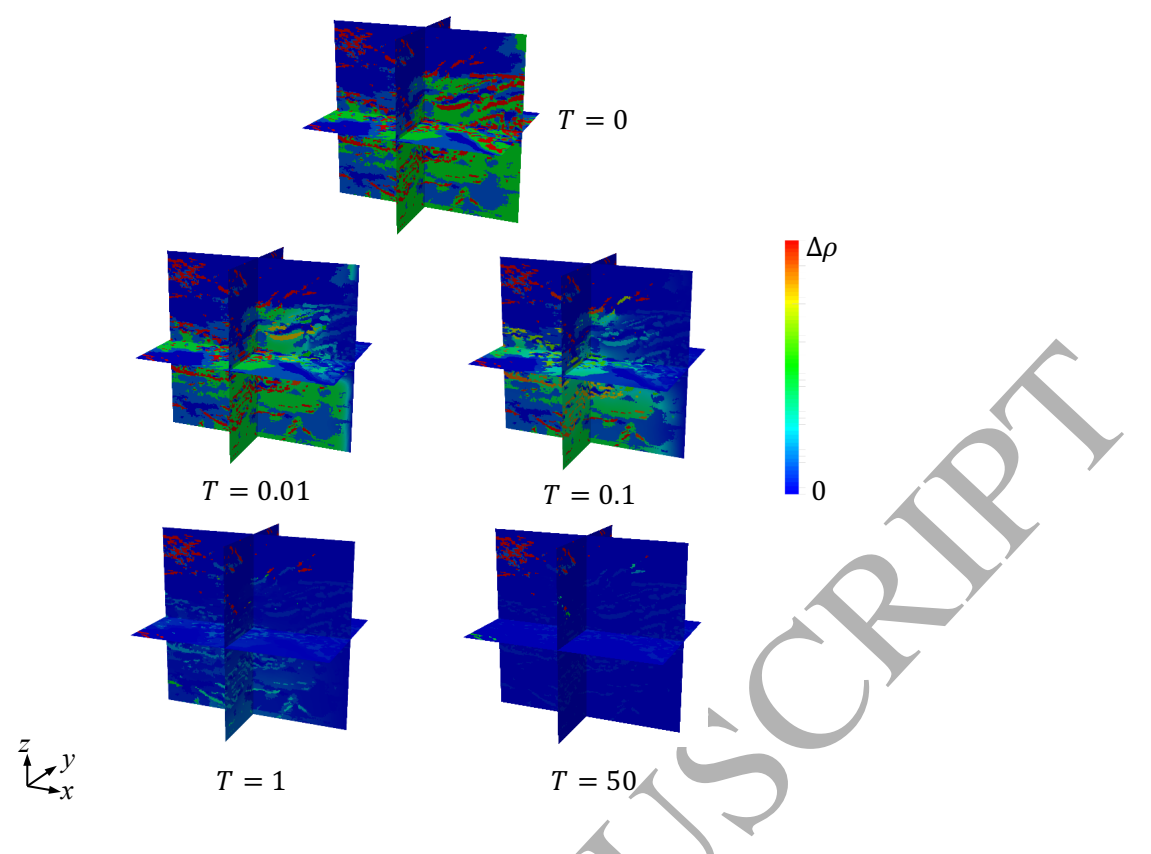

Figure 12: $3 \mathrm{D}$ visualization of gas production at $T=0,0.01,0.1,1,50$ for low pressure $(p=1 \mathrm{MPa})$ using the full model. The colour represents the remaining producible gas per voxel due to pressure change at the right boundary. Note that the producible gas in organic matter includes both free gas and adsorbed gas.

\section{Discussions}

The simulation results from the micro-continuum model consider both the steady-state apparent permeability and the transient gas production process. The apparent permeability for shale has been a focus of many recent experimental and modeling work. Pressure pulse-decay method, a technique designed to measure permeability of tight rocks $[51,52,54]$, has been used to measure the apparent permeability of shale core plugs [e.g., 53, 55, 56]. This is done by fitting a $1 \mathrm{D}$ model (similar to the $1 \mathrm{D}$ model in section 4.3 .1 when non-Darcy effects, adsorption/desorption, and surface diffusion are not included) to the pressure curve from the dynamically changing flow due to the pressure pulse. The pressure pulse-decay method works well for conventional tight rocks, but whether it can be directly applied to the much more complex organic-rich shale is still an open question [53,57]. Various models have been developed to understand how the transport mechanisms (non-Darcy effects, adsorption/desorption, surface diffusion) control the apparent permeability of shale. For example, bundle-of-tubes models [e.g., 19, 24]), pore network models (PNM) [e.g., 58, 59], and lattice Boltzmann simulations [e.g., 33]. These models either consider idealized or approximated pore structures (bundle-of-tubes model and PNM models) or only consider the resolved pores and neglect the sub-resolution nano-pore structures (LBM simulations). Our micro-continuum model offers a direct reference solution of gas transport in 3D real digital images of shale that considers both the resolved macro-pores and sub-resolution pore structures. Demonstrated by the two numerical experiments, the micro-continuum model can be used to compute the steady-state apparent permeability as well as analyze the transient be- 
havior of gas transport using a pressure decay setup. The direct reference solutions of gas transport in 3D shale images may provide new insights into the interpretation of pressure pulse-decay experiments shown by the transient analysis in the second numerical experiment.

The micro-continuum modeling framework is able to model gas transport on a high-resolution 3D digital shale image including non-Darcy effects, adsorption/desorption, which offers a quantitative tool to analyze the transport properties of shale. Nevertheless, there are a few challenges that need to be addressed to improve the modeling framework. For example, higher resolution images that can resolve the pores in the subresolution nano-porous regions can be helpful to better estimate the intrinsic and apparent permeabilities of the organic matter and clay. When the Stokes flow in macro-pores falls into the slip flow regime, appropriate velocity slip conditions at the wall needs to be developed. The current model has non-zero velocities at the boundaries between macro-pores and the nano-porous regions (organic matter and clay), but the boundaries between macro-pores and the granular minerals are considered as no-slip. Finally, the current computational algorithm based on the Darcy-Brinkman-Stokes framework turns out to be very computationally expensive, which forces us to use a subset of the original full image to reduce the computational cost. Even for the sub-image, it takes a few days to a week to finish one simulation using about 100 processors on a computer cluster. It is therefore desirable to speed up the micro-continuum model, e.g., by developing multiscale algorithms, which is part of our ongoing research.

\section{Conclusion}

We have developed a micro-continuum pore-scale modeling framework for gas transport in organic-rich shale using high-resolution 3D digitalimages. We model flow in the resolved macro-pores using the Stokes equation; we consider the sub-resolution nano-porous region (organic matter or clay) as a continuum, and develop an apparent permeability model to include the non-Darcy effects (e.g., slip flow and Knudsen diffusion). Adsorption/desorption and surface diffusion are included in the sub-resolution organic matter region. This modeling framework allows us to investigate the complex transport mechanisms involved in organic-rich shale formations. We apply the micro-continuum model to simulate a 3D FIB-SEM image using two different settings. The first setting allows for computing an apparent permeability, and the other setting simulates the transient gas production process. Our simulation results show that non-Darcy and surface diffusion effects are important at low gas pressure $(1 \mathrm{MPa})$; these mechanisms become negligible at high pressure (50 MPa). Our gas production analysis indicates that a simple pressure dependent apparent permeability may not represent the transport properties of organic-rich shale. Depending on the pore structures of the shale image, different time scales may need to be introduced to better describe the transport properties of shale at a fixed gas pressure. 


\section{Acknowledgement}

The image datasets were provided by L. Ma, P. D. Lee and K. Taylor and generated through funding from NERC-UK (NE/M001458/1), the Research Complex at Harwell, and the European Union Horizon 2020716 Research and Innovation Program under the ShaleXenvironmenT project, (grant no. 640979). We thank C. Soulaine for discussions on OpenFOAM and Y. Mehmani for discussions on image processing. This work is supported in part by TOTAL through the Stanford TOTAL enhanced modeling of source rock (STEMS) project.

\section{Appendix A. Equation-of-state and viscosity equation for methane}

We use the empirical correlation from Mahmoud [38] as the equation of state for methane, and follow the empirical equation from Lee et al. [39] to compute viscosity. The empirical correlation from Mahmoud [38] is derived using the compressibility factor approach. Here, we outline the empirical equations for density and viscosity respectively.

We rescale the pressure and temperature by the critical pressure and temperature and define the dimensionless reduced pressure and temperature

$$
\begin{aligned}
p_{f, r} & =\frac{p_{f}}{p_{f, \text { critical }}}, \\
T_{r} & =\frac{T}{T_{\text {critical }}},
\end{aligned}
$$

where $p_{f}$ and $T$ are the absolute pressure and temperature of free methane; $p_{f, \text { crtical }}$ and $T_{\text {critical }}$ are the critical pressure and temperature. Then, the empirical correlation for the compressibility factor, $Z$, has the following form

$$
Z=0.702 e^{-2.5 T_{f, r}} p_{f, r}^{2}-5.524 e^{-2.5 T_{f, r}} p_{f, r}+0.044 T_{f, r}^{2}-0.164 T_{f, r}+1.15 \text {. }
$$

Substituting $Z$ to Equation (1) gives the density of methane. After we obtain density, we can compute viscosity using the equations from Lee et al. [39].

$$
\begin{gathered}
\mu_{f}=10^{-7} S e^{X\left(0.001 \rho_{f}\right)^{Y}}, \\
S=\frac{(9.379+0.0160 M)(1.8 T)^{1.5}}{209.2+19.26 M+1.8 T}, \\
X=3.448+\frac{986.4}{1.8 T}+0.01009 M,
\end{gathered}
$$




$$
Y=2.4-0.2 X \text {. }
$$

$\mu_{f}, \rho_{f}, M, T$ in Equations (A.4), (A.5) and (A.6) all have SI base units. Note: (1) some of the coefficient constants in Equations (A.4), (A.5) and (A.6) are modified from Lee et al. [39] based on Equation (62) in Mahmoud [38]; (2) the temperature in Lee et al. [39] is in Rankine scale $\left({ }^{\circ} \mathrm{R}\right)$ and we converted it to Kelvin scale $(\mathrm{K})$ by multiplying by 1.8. We have compared the density and viscosity of free methane with the methane density and viscosity from the NIST fluid database [60], and we observe a maximum relative error less than $5 \%$.

\section{Appendix B. Scaling arguments for Equation (15)}

Here we present a scaling argument to show that the momentum equation for compressible flow can be simplified to the form of Equation (15). We define the following dimensionless variables and groups

$$
\begin{gathered}
\widetilde{\mathbf{x}}=\frac{\mathbf{x}}{l_{c}}, \widetilde{t}=\frac{t}{t_{c}}, \widetilde{\mathbf{u}}_{f}=\frac{\mathbf{u}_{f}}{l_{c} / t_{c}}, \widetilde{p}_{f}=\frac{p_{f}}{\mu_{c} u_{c} / l_{c}}, \widetilde{\mathbf{g}} \neq \frac{\mathbf{g}}{g}, \widetilde{\rho}_{f}=\frac{\rho_{f}}{\rho_{f, c}}, \widetilde{\mu}_{f}=\frac{\mu_{f}}{\mu_{f, c}}, \\
\operatorname{Re}=\frac{\rho_{f, c} u_{f, c} l_{c}}{\mu_{f, c}}, \operatorname{Fr}=\frac{g l_{c}}{u_{f, c}^{2}},
\end{gathered}
$$

where $u_{f, c}, p_{f, c}$ are characteristic velocity and pressure; $l_{c}$ and $t_{c}$ are characteristic length and time scales; $\mu_{f, c}$ and $\rho_{f, c}$ are characteristic viscosity and density; Re is the Reynolds number and Fr is the Froude number that defines the relative importance of gravity and viscosity effects.

Substituting the nondimensionalized variables to Equation (2.15) with the time derivative term, we obtain

$$
\operatorname{Re} \frac{\partial\left(\widetilde{\rho}_{f} \widetilde{\mathbf{u}}_{f}\right)}{\partial \widetilde{t}}=-\nabla \widetilde{p}_{f}+\frac{\operatorname{Re}}{\operatorname{Fr}} \widetilde{\rho}_{f} \widetilde{\mathbf{g}}+\nabla \cdot\left(\widetilde{\mu}_{f} \nabla \widetilde{\mathbf{u}}_{f}\right)+\frac{1}{3} \nabla\left(\widetilde{\mu}_{f} \nabla \cdot \widetilde{\mathbf{u}}_{f}\right)
$$

For the extremely tight shale rock with nanometer-scale pore structures, the Reynolds number Re $\ll 1$, which means that the time derivative term is eligible compared to the terms related to the pressure gradient and the viscous forces

\section{Appendix C. Numerical algorithm for the micro-continuum model}

We introduce the numerical algorithm to solve Equations (16) and (17) based on the PIMPLE algorithm in OpenFOAM. Discretization of the equations here follows in part chapter 3 of Jasak [61], which presents discretizations of the Navier-Stokes equations for incompressible flow. 


\section{Discretization}

The PIMPLE algorithm solves velocity and pressure implicitly through sequentially coupling. Here, we derive the discretized forms of the momentum and pressure equations.

Discretizing the integral form of the momentum Equation (17) over a control volume $V$ with backward Euler time stepping gives

$$
a_{P}^{\mathrm{n}+1} \mathbf{u}_{P}^{\mathrm{n}+1}=\sum_{N P} a_{N P}^{\mathrm{n}+1} \mathbf{u}_{N P}^{\mathrm{n}+1}-\nabla p^{\mathrm{n}+1}+\rho_{f}^{\mathrm{n}+1} \mathbf{g},
$$

where both sides of the equation are divided by the control volume $V . \mathbf{u}_{P}$ is the cell-centered velocity of the current cell, and $\mathbf{u}_{N P}$ denotes the cell-centered velocity of the neighbouring cells; subscripts ' $P$ ' and ' $N P$ ' denote the current cell and neighbouring cells, respectively. $a_{P}^{\mathrm{n}+1}$ consists coefficients contributed by the divergence term $\left(\nabla \cdot\left(\frac{\mu_{f}}{\phi} \nabla \mathbf{u}_{f}\right)\right)$ and the apparent Darcy term $\mu_{f} k_{a}^{-1} \mathbf{u}_{f}$, while $a_{N P}^{\mathrm{n}+1}$ only has coefficients from the divergence term. The superscript ' $n$ ' and ' $n+1$ ' denote time steps. We note that the pressure gradient term and the gravity term are not discretized in space at this stage.

Now we formulate the pressure equation based on the semi-discretized form of the momentum Equation (C.1). We divide both sizes of Equation (C.1) by $a_{P}$ and interpolate the equation at the cell face, and obtain

$$
\begin{aligned}
\left(\mathbf{u}_{P}^{\mathrm{n}+1}\right)_{\text {face }}= & \left(\frac{1}{a_{P}^{\mathrm{n}+1}}\right)_{\text {face }}\left(\sum_{N P} a_{N P}^{\mathrm{n}+1} \mathbf{u}_{N P}^{\mathrm{n}+1}\right)_{\text {face }}-\left(\frac{1}{a_{P}^{\mathrm{n}+1}}\right)_{\text {face }}\left(\nabla p^{\mathrm{n}+1}\right)_{\text {face }} \\
& +\left(\frac{1}{a_{P}^{\mathrm{n}+1}}\right)_{\text {face }}\left(\rho_{f}^{\mathrm{n}+1} \mathbf{g}\right)_{\text {face }} .
\end{aligned}
$$

Substituting Equation (C.2) into the integral form of the mass balance Equation (16), we obtain the pressure equation

$$
\begin{aligned}
A_{1}^{\mathrm{n}+1} \frac{p^{\mathrm{n}+1}-p^{\mathrm{n}}}{\Delta t}-\sum_{\text {face }} S \cdot\left[A_{2}^{\mathrm{n}+1}\left(\nabla p^{\mathrm{n}+1}\right)_{\text {face }}\right] & +\sum_{\text {face }} S \cdot\left[A_{3}^{\mathrm{n}+1}\left(\sum_{N P} a_{N P}^{\mathrm{n}+1} \mathbf{u}_{N P}^{\mathrm{n}+1}\right)_{\text {face }}\right] \\
& +\sum_{\text {face }} S \cdot\left[A_{3}^{\mathrm{n}+1}\left(\rho_{f}^{\mathrm{n}+1} \mathbf{g}\right)_{\text {face }}\right]=0,
\end{aligned}
$$
$\left(\delta_{\text {om }} D_{s} n_{\text {ad }}^{\max } \frac{k}{(1+K p)^{2}}\right)_{\text {face }}, A_{3}=\left(\rho_{f}\right)_{\text {face }}\left(\frac{1}{a_{P}}\right)_{\text {face }}$.

Now we need to solve the discretized momentum Equation (C.1) and pressure Equation (C.3).

\section{Solution procedure}

We introduce the solution procedure to solve the discrete momentum and pressure Equations (C.1) and (C.3) following the PIMPLE algorithm. The momentum and pressure equations are solved sequentially in PIMPLE, and iterations are required between the velocity and pressure solutions within one time step to reach convergence before moving on to the next time step. The momentum-pressure iterations are similar 
to the SIMPLE algorithm, and are referred to as outer correction loops. Within each momentum-pressure correction loop, pressure can be solved multiple times followed by explicit velocity corrections after the implicit solution of the momentum equation. The pressure and velocity corrections within a momentumpressure correction loop are referred to as inner corrections, which is similar to the PISO algorithm. Here we outline the solution procedure from time step $n$ to time step $n+1$, and from outer correction loop i to $\mathrm{i}+1$. The inner corrections are described in words.

1. Solve momentum Equation (C.1). This step is called momentum predictor. The pressure gradient is computed using pressure distribution from the outer correction loop $i$, and the velocity is under-relaxed in an implicit manner as the following

$$
\begin{aligned}
a_{P}^{\mathrm{n}+1, \mathrm{i}} \mathbf{u}_{P}^{\mathrm{n}+1, \mathrm{i}+1, *}+\frac{1-\alpha_{u}}{\alpha_{u}} \mathbf{u}_{P}^{\mathrm{n}+1, \mathrm{i}+1, *}= & \sum_{N P} a_{N P}^{\mathrm{n}+1, \mathrm{i}} \mathbf{u}_{N P}^{\mathrm{n}+1, \mathrm{i}+1, *}-\nabla p^{\mathrm{n}+1, \mathrm{i}}+\rho_{f} \mathbf{g} \\
& +\frac{1-\alpha_{u}}{\alpha_{u}} a_{P}^{\mathrm{n}+1, \mathrm{i}} \mathbf{u}_{P}^{\mathrm{n}+1, \mathrm{i}},
\end{aligned}
$$

where $\alpha_{u}$ is the under-relaxation factor for velocity. We denote the updated velocity with a superscript '*' because it is an approximation with the pressure field from the previous outer correction loop.

2. Solve pressure Equation (C.3) with updated velocity $\mathbf{u}^{n+1, i+1, *}$ from step 1. Then update velocity in an explicit manner following Equation (C.2) using the new pressure, and solve pressure Equation (C.3) again. This is called inner correction. We repeat the inner/correction until we reach the specified number of inner corrections.

3. Under relax the pressure solution based on Equation (C.5)

$$
=p^{\mathrm{n}+1, \mathrm{i}}+\alpha_{p}\left(p^{\mathrm{n}+1, \mathrm{i}+1, *}-p^{\mathrm{n}+1, \mathrm{i}}\right)
$$

where $p^{\mathrm{n}+1, \mathrm{i}}$ is the pressure from the previous outer correction loop $i, p^{\mathrm{n}+1, \mathrm{i}+1, *}$ is the pressure obtained after step $2, p^{\mathrm{n}+1, \mathrm{i}+1}$ is the pressure that will be used in the next momentum predictor to start the next outer correction loop, and $\alpha_{p}$ is the under-relaxation factor for pressure.

4. Repeat steps 1-3 for the next outer correction loop until reaching a specified number of outer correction loops or specified residual tolerance for velocity and pressure.

5. Repeat steps 1-4 for the next time step until reaching the end of the simulation time.

\section{Appendix D. Derivations of the 1D model in section 4.3.1}

Here we present the details to derive Equation (23) from Equation (22).

The first term of Equation (22)

$$
\frac{\partial\left(\bar{\phi} \rho_{f}\right)}{\partial t}=\bar{\phi} \frac{\partial \rho_{f}}{\partial t}=\bar{\phi} \frac{\partial \rho_{f}}{\partial p_{f}} \frac{\partial p_{f}}{\partial t}
$$


The second term of Equation (22)

$$
\bar{\gamma}_{\mathrm{om}} \frac{\partial n_{\mathrm{ad}}}{\partial t}\left(1-\frac{\rho_{f}}{\rho_{\mathrm{ad}}}\right)=\bar{\gamma}_{\mathrm{om}} n_{\mathrm{ad}}^{\max } \frac{K}{\left(1+K p_{f}\right)^{2}}\left(1-\frac{\rho_{f}}{\rho_{\mathrm{ad}}}\right) \frac{\partial p_{f}}{\partial t} .
$$

653

Putting together all three terms of Equation (22), we get

$$
\begin{array}{r}
\left(\bar{\phi} \frac{\partial \rho_{f}}{\partial p_{f}}+\bar{\gamma}_{\mathrm{om}} n_{\mathrm{ad}}^{\max } \frac{K}{\left(1+K p_{f}\right)^{2}}\left(1-\frac{\rho_{f}}{\rho_{\mathrm{ad}}}\right)\right) \frac{\partial p_{f}}{\partial t} \\
=\left(\rho_{f} \frac{\bar{k}_{a}}{\mu_{f}}+\bar{\gamma}_{\mathrm{om}} \rho_{\mathrm{ad}} D_{s} n_{\mathrm{ad}}^{\max } \frac{K}{\left(1+K p_{f}\right)^{2}}\right) \frac{\partial^{2} p_{f}}{\partial x^{2}}
\end{array}
$$

The third term of Equation (22)

$$
\begin{aligned}
\frac{\partial\left(\rho_{f} u_{f, x}+\bar{\gamma}_{\mathrm{om}} \rho_{\mathrm{ad}} u_{\mathrm{ad}, x}\right)}{\partial x}= & \frac{\partial\left(\rho_{f} u_{f, x}\right)}{\partial x}+\frac{\partial\left(\bar{\gamma}_{\mathrm{om}} \rho_{\mathrm{ad}} u_{\mathrm{ad}, x}\right)}{\partial x} \\
= & -\rho_{f} \frac{\bar{k}_{a}}{\mu_{f}} \frac{\partial^{2} p_{f}}{\partial x^{2}}-\bar{\gamma}_{\mathrm{om}} \rho_{\mathrm{ad}} D_{s} n_{\mathrm{ad}} \max \frac{\partial}{\partial x}\left[\frac{K}{\left(1+K p_{f}\right)^{2}} \frac{\partial p_{f}}{\partial x}\right] \\
= & -\rho_{f} \frac{\bar{k}_{a}}{\mu_{f}} \frac{\partial^{2} p_{f}}{\partial x^{2}} \\
& -\bar{\gamma}_{\mathrm{om}} \rho_{\mathrm{ad}} D_{s} n_{\mathrm{ad}}^{\max } \frac{K}{\left(1+K p_{f}\right)^{2}} \frac{\partial^{2} p_{f}}{\partial x^{2}} \\
& \left.-\bar{\gamma}_{\mathrm{om}} \rho_{\mathrm{ad}} D_{s} n_{\mathrm{ad}}^{\max } \frac{2 K^{2}}{\left(1+K p_{f}\right)^{3}}\left(\frac{\partial p_{f}}{\partial x}\right)^{2}\right)
\end{aligned}
$$

Now we show that $\frac{2 K^{2}}{\left(1+K p_{f}\right)^{3}}\left(\frac{\partial p_{f}}{\partial x}\right)^{2} \ll \frac{K}{\left(1+K p_{f}\right)^{2}} \frac{\partial^{2} p_{f}}{\partial x^{2}}$. These two terms have the following scaling

$$
\begin{aligned}
\frac{2 K^{2}}{\left(1+K p_{f}\right)^{3}}\left(\frac{\partial p_{f}}{\partial x}\right)^{2} & \sim \frac{2 K^{2}}{\left(1+K p_{f}\right)^{3}}\left(\frac{\Delta p}{L_{x}}\right)^{2}, \\
\frac{K}{\left(1+K p_{f}\right)^{2}} \frac{\partial^{2} p_{f}}{\partial x^{2}} & \sim \frac{\Delta}{\left(1+K p_{f}\right)^{2}} \frac{\Delta p}{L_{x}^{2}} .
\end{aligned}
$$

We take a ratio of the two terms, and obtain $\left[\frac{2 K^{2}}{\left(1+K p_{f}\right)^{3}}\left(\frac{\Delta p}{L_{x}}\right)^{2}\right] /\left[\frac{K}{\left(1+K p_{f}\right)^{2}} \frac{\Delta p}{L_{x}^{2}}\right]=\frac{2 K \Delta p}{1+K p_{f}}<2 K \Delta p=$ $8 \times 10^{-6} \ll 1$. Therefore, $\frac{2 K^{2}}{\left(1+K p_{f}\right)^{3}}\left(\frac{\partial p_{f}}{\partial x}\right)^{2}$ can be neglected, so the third term of Equation $(22)$ can be approximated as

$$
\frac{\partial\left(\rho_{f} u_{f, x}+\bar{\gamma}_{\mathrm{om}} \rho_{\mathrm{ad}} u_{\mathrm{ad}, x}\right)}{\partial x} \approx-\rho_{f} \frac{\bar{k}_{a}}{\mu_{f}} \frac{\partial^{2} p_{f}}{\partial x^{2}}-\bar{\gamma}_{\mathrm{om}} \rho_{\mathrm{ad}} D_{s} n_{\mathrm{ad}}^{\max } \frac{K}{\left(1+K p_{f}\right)^{2}} \frac{\partial^{2} p_{f}}{\partial x^{2}} .
$$

Rearranging Equation (D.7) gives the Equation (23).

\section{References}

[1] EIA, Natural gas annual report, U.S. Energy Information Administration (2017). 
[2] D. J. Ross, R. M. Bustin, The importance of shale composition and pore structure upon gas storage potential of shale gas reservoirs, Marine and Petroleum Geology 26 (2009) 916-927.

[3] Y. Gensterblum, A. Ghanizadeh, R. J. Cuss, A. Amann-Hildenbrand, B. M. Krooss, C. R. Clarkson, J. F. Harrington, M. D. Zoback, Gas transport and storage capacity in shale gas reservoirs-a review. part a: Transport processes, Journal of Unconventional Oil and Gas Resources 12 (2015) 87-122.

[4] R. W. Edwards, M. A. Celia, K. W. Bandilla, F. Doster, C. M. Kanno, A model to estimate carbon dioxide injectivity and storage capacity for geological sequestration in shale gas wells, Environmental science \& technology 49 (2015) 9222-9229.

[5] C. H. Sondergeld, R. J. Ambrose, C. S. Rai, J. Moncrieff, Micro-structural studies of gas shales, in: SPE Unconventional Gas Conference, Society of Petroleum Engineers, 2010.

[6] G. R. Chalmers, R. M. Bustin, I. M. Power, Characterization of gas shale pore systems by porosimetry, pycnometry, surface area, and field emission scanning electron microscopy/transmission electron microscopy image analyses: Examples from the barnett, woodford, haynesville, marcellus, and doig units, AAPG bulletin 96 (2012) 1099-1119.

[7] C. R. Clarkson, N. Solano, R. M. Bustin, A. Bustin, G. Chalmers, L. He, Y. B. Melnichenko, A. Radliński, T. P. Blach, Pore structure characterization of north american shale gas reservoirs using usans/sans, gas adsorption, and mercury intrusion, Fuel 103 (2013) 606-616.

[8] M. E. Curtis, C. H. Sondergeld, R. J. Ambrose, C. S. Rai, Microstructural investigation of gas shales in two and three dimensions using nanometer-scale resolution imaging, AAPG bulletin 96 (2012) 665-677.

[9] L. Ma, A.-L. Fauchille, P.J. Dowey, F. F. Pilz, L. Courtois, K. G. Taylor, P. D. Lee, Correlative multi-scale imaging of shales: a review and future perspectives, Geological Society, London, Special Publications 454 (2017) 175-199.

[10] S. Kelly, H. E1-Sobky, C. Torres-Verdín, M. T. Balhoff, Assessing the utility of fib-sem images for shale digital rock physics, Advances in Water Resources 95 (2016) 302-316.

[11] L. Ma, K. G. Taylor, P. D. Lee, K. J. Dobson, P. J. Dowey, L. Courtois, Novel 3d centimetre-to nanoscale quantification of an organic-rich mudstone: The carboniferous bowland shale, northern england, Marine and Petroleum Geology 72 (2016) 193-205.

[12] L. Ma, T. Slater, P. J. Dowey, S. Yue, E. H. Rutter, K. G. Taylor, P. D. Lee, Hierarchical integration of porosity in shales, Scientific reports 8 (2018) 11683.

[13] T. Wu, X. Li, J. Zhao, D. Zhang, Mutiscale pore structure and its effect on gas transport in organic-rich shale, Water Resources Research 53 (2017) 5438-5450. 
[14] J. Rouquerol, D. Avnir, C. Fairbridge, D. Everett, J. Haynes, N. Pernicone, J. Ramsay, K. Sing, K. Unger, Recommendations for the characterization of porous solids (technical report), Pure and Applied Chemistry 66 (1994) 1739-1758.

[15] L. Klinkenberg, The permeability of porous media to liquids and gases, in: Drilling and production practice, American Petroleum Institute, 1941.

[16] G. E. Karniadakis, A. Beskok, N. Aluru, Microflows and Nanoflows: Fundamentals and Simulation, Springer, 2005.

[17] A. Beskok, G. E. Karniadakis, Report: a model for flows in channels, pipes, and ducts át micro and nano scales, Microscale Thermophysical Engineering 3 (1999) 43-77.

[18] T. Wu, D. Zhang, Impact of adsorption on gas transport in nanopores, Scientific reports 6 (2016) 23629.

[19] F. Civan, Effective correlation of apparent gas permeability in tight porous media, Transport in porous media 82 (2010) 375-384.

[20] X. Xiong, D. Devegowda, M. Villazon, G. German, R. F. Sigal, F. Civan, A fully-coupled free and adsorptive phase transport model for shale gas reservoirs including non-darcy flow effects, in: SPE annual technical conference and exhibition, Society of Petroleum Engineers, 2012.

[21] I. Lunati, S. Lee, A dual-tube model for gas dynamics in fractured nanoporous shale formations, Journal of Fluid Mechanics 757 (2014) 943-971.

[22] E. A. Mason, A. Malinauskas, Gas transport in porous media: the dusty-gas model, volume 17, Elsevier Science Ltd, 1983.

[23] F. Javadpour, Nanopores and apparent permeability of gas flow in mudrocks (shales and siltstone), Journal of Canadian Petroleum Technology 48 (2009) 16-21.

[24] H. Darabi, A.Ettehad, F. Javadpour, K. Sepehrnoori, Gas flow in ultra-tight shale strata, Journal of Fluid Mechanics 710 (2012) 641-658.

[25] A. Sakhaee-Pour, S. Bryant, Gas permeability of shale, SPE Reservoir Evaluation \& Engineering 15 (2012) $401-409$.

[26] K. Wu, X. Li, C. Guo, C. Wang, Z. Chen, A unified model for gas transfer in nanopores of shale-gas reservoirs: coupling pore diffusion and surface diffusion, SPE Journal 21 (2016) 1-583.

[27] C. J. Landry, M. Prodanović, P. Eichhubl, Direct simulation of supercritical gas flow in complex nanoporous media and prediction of apparent permeability, International Journal of Coal Geology 159 (2016) 120-134. 
[28] I. Y. Akkutlu, Y. Efendiev, V. Savatorova, Multi-scale asymptotic analysis of gas transport in shale matrix, Transport in Porous Media 107 (2015) 235-260.

[29] M. J. Blunt, B. Bijeljic, H. Dong, O. Gharbi, S. Iglauer, P. Mostaghimi, A. Paluszny, C. Pentland, Pore-scale imaging and modelling, Advances in Water Resources 51 (2013) 197-216.

[30] M. J. Blunt, Multiphase flow in permeable media: A pore-scale perspective, Cambridge University Press, 2017.

[31] J. Wang, L. Chen, Q. Kang, S. S. Rahman, The lattice boltzmann method for isothermal micro-gaseous flow and its application in shale gas flow: a review, International Journal of Heat and Mass Transfer 95 (2016) 94-108.

[32] L. Chen, L. Zhang, Q. Kang, H. S. Viswanathan, J. Yao, W. Tao, Nanoscale simulation of shale transport properties using the lattice boltzmann method: permeability and diffusivity, Scientific reports 5 (2015).

[33] J. Wang, Q. Kang, L. Chen, S. S. Rahman, Pore-scale lattice boltzmann simulation of micro-gaseous flow considering surface diffusion effect, International Journal of Coal Geology 169 (2017) 62-73.

[34] C. Soulaine, H. A. Tchelepi, Micro-continuum approach for pore-scale simulation of subsurface processes, Transport in Porous Media 113 (2016) 431-456.

[35] H. Brinkman, A calculation of the viscous force exerted by a flowing fluid on a dense swarm of particles, Applied Scientific Research 1 (1949) 27-34.

[36] A. Mehmani, M. Prodanović, The effect of microporosity on transport properties in porous media, Advances in Water Resources 63 (2014) 104-119.

[37] T. Bultreys, L. Van Hoorebeke, V.Cnudde, Multi-scale, micro-computed tomography-based pore network models to simulate drainage in heterogeneous rocks, Advances in Water Resources 78 (2015) $36-49$.

[38] M. Mahmoud, Development of a new correlation of gas compressibility factor (z-factor) for high pressure gas reservoirs, Journal of Energy Resources Technology 136 (2014) 012903.

[39] A. L. Lee, M. H. Gonzalez, B. E. Eakin, The viscosity of natural gases, Journal of Petroleum Technology 18 (1966) 997-1.

[40] D., M. Ruthven, Principles of adsorption and adsorption processes, John Wiley \& Sons, 1984.

[41] I. Medved̆, R. Černỳ, Surface diffusion in porous media: A critical review, Microporous and Mesoporous Materials 142 (2011) 405-422.

[42] R. Heller, M. Zoback, Adsorption of methane and carbon dioxide on gas shale and pure mineral samples, Journal of Unconventional Oil and Gas Resources 8 (2014) 14-24. 
[43] T. F. Rexer, E. J. Mathia, A. C. Aplin, K. M. Thomas, High-pressure methane adsorption and characterization of pores in posidonia shales and isolated kerogens, Energy \& Fuels 28 (2014) 2886-2901.

[44] T. Zhang, G. S. Ellis, S. C. Ruppel, K. Milliken, R. Yang, Effect of organic-matter type and thermal maturity on methane adsorption in shale-gas systems, Organic Geochemistry 47 (2012) 120-131.

[45] S. Bakhshian, Z. Shi, M. Sahimi, T. T. Tsotsis, K. Jessen, Image-based modeling of gas adsorption and deformation in porous media, Scientific reports 8 (2018) 8249.

[46] G. S. Beavers, D. D. Joseph, Boundary conditions at a naturally permeable wall, Journal of fluid mechanics 30 (1967) 197-207.

[47] B. Goyeau, D. Lhuillier, D. Gobin, M. Velarde, Momentum transport at a fluid-porous interface, International Journal of Heat and Mass Transfer 46 (2003) 4071-4081.

[48] T. D. Scheibe, W. A. Perkins, M. C. Richmond, M. I. McKinley, P. D. Romero-Gomez, M. Oostrom, T. W. Wietsma, J. A. Serkowski, J. M. Zachara, Pore-scale and multiscale numerical simulation of flow and transport in a laboratory-scale column, Water Resources Research 51 (2015) 1023-1035.

[49] R. I. Issa, Solution of the implicitly discretised fluid flow equations by operator-splitting, Journal of computational physics 62 (1986) 40-65.

[50] S. Patankar, Numerical heat transfer and fluid flow, CRC press, 1980.

[51] W. Brace, J. Walsh, W. Frangos, Permeability of granite under high pressure, Journal of Geophysical research 73 (1968) 2225-2236.

[52] A. Dicker, R. Smits, A practical approach for determining permeability from laboratory pressurepulse decay measurements, in: International meeting on petroleum engineering, Society of Petroleum Engineers, 1988.

[53] X. Cui, A. Bustin, R. M. Bustin, Measurements of gas permeability and diffusivity of tight reservoir rocks: different approaches and their applications, Geofluids 9 (2009) 208-223.

[54] S. Jones, A technique for faster pulse-decay permeability measurements in tight rocks, SPE formation evaluation 12 (1997) 19-26.

[55] R. Heller, J. Vermylen, M. Zoback, Experimental investigation of matrix permeability of gas shales, AAPG bulletin 98 (2014) 975-995.

[56] B. Abdelmalek, Z. Karpyn, S. Liu, H. Yoon, T. Dewers, Gas permeability measurements from pressure pulse decay laboratory data using pseudo-pressure and pseudo-time transformations, Journal of Petroleum Exploration and Production Technology (2017) 1-9. 
[57] K. R. Alnoaimi, C. Duchateau, A. R. Kovscek, Characterization and measurement of multiscale gas transport in shale-core samples, SPE Journal 21 (2016) 573-588.

[58] A. Mehmani, M. Prodanović, F. Javadpour, Multiscale, multiphysics network modeling of shale matrix gas flows, Transport in porous media 99 (2013) 377-390.

[59] W. Song, J. Yao, J. Ma, G. Couples, Y. Li, Assessing relative contributions of transport mechanisms and real gas properties to gas flow in nanoscale organic pores in shales by pore network modelling, International Journal of Heat and Mass Transfer 113 (2017) 524-537.

[60] E. Lemmon, M. McLinden, D. Friend., Thermophysical properties of fluid systems, in: P. Linstrom, W. Mallard (Eds.), NIST Chemistry WebBook, NIST Standard Reference Database Number 69, National Institute of Standards and Technology, Gaithersburg MD, 1998.

[61] H. Jasak, Error Analysis and Estimation for the Finite Volume Method with Applications to Fluid Flows, 1996, Ph.D. thesis, Ph. D. Thesis, University of London Imperial College, 1996. 